Article

\title{
Chiral Brønsted acid-catalyzed conjugate addition of indoles to azadienes: Enantioselective synthesis of hetero-triarylmethanes
}

\author{
Huan-Ping Xie a,b, Bo Wu a , Xin-Wei Wang a, Yong-Gui Zhou a,* \\ a State Key Laboratory of Catalysis, Dalian Institute of Chemical Physics, Chinese Academy of Sciences, Dalian 116023, Liaoning, China \\ b University of Chinese Academy of Sciences, Beijing 100049, China
}

\section{A R T I C L E I N F O}

\section{Article history:}

Received 27 June 2019

Accepted 20 July 2019

Published 5 October 2019

Keywords:

Conjugate addition

Hetero-triarylmethane

Azadiene

Indole

Chiral Brønsted acid

\begin{abstract}
A B S T R A C T
An efficient chiral Brønsted acid-catalyzed conjugate addition of indoles to azadienes has been successfully developed, which enables a facile approach to optically active hetero-triarylmethanes with excellent enantioselectivities and broad substrate scope. This chiral Brønsted acid catalytic system provides a new opportunity for the development of asymmetric reactions of azadienes.
\end{abstract}

(C) 2019, Dalian Institute of Chemical Physics, Chinese Academy of Sciences. Published by Elsevier B.V. All rights reserved.

\section{Introduction}

Aurone-derived azadienes have been regarded as a pivotal class of highly reactive intermediates in organic synthesis due to the driving force of aromatization [1-20]. In the past few years, only a few catalytic asymmetric processes of azadienes have been successfully developed on the basis of transition-metal catalysis and organo-catalysis [10-20]. Zhao and co-workers [10-12] disclosed palladium-catalyzed asymmetric formal cycloaddition of azadienes to prepare medium-sized compounds including benzofuran- fused nine-membered and ten-membered heterocycles. Organocatalytic systems for the asymmetric reactions of azadienes mainly focused on chiral amine, $N$-heterocyclic carbene (NHC), phosphine and bifunctional Brønsted base. In 2016, Zhao's group [13] reported chiral amine-catalyzed aza-Diels-Alder reactions of azadienes with aldehydes to afford tetrahydropyridines and NHC-catalyzed
aza-Diels-Alder reactions of azadienes with $\alpha$-chloroaldehydes to provide benzofuran-fused lactams. The catalytic systems, chiral amine and NHC, are complementary and made it possible to achieve diastereodivergent and highly stereoselective transformations. Ye and co-workers [14,15] developed NHC-catalyzed [4+3] annulation of azadienes with acyclic enals to deliver benzofuroazepinones with excellent stereoselectivities. The enantioselective amino-acid-derived phosphine-catalyzed formal [4+4] cycloaddition of azadienes with allene ketones to afford eight-membered structural motifs has been established by Lu's group [16]. Recently, chiral Brønsted bases have been used as highly enantioselective bifunctional catalysts for the asymmetric nucleophilic addition of phosphites, thiols and rhodanines to azadienes [17-19]. Additionally, the formal [4+2] cycloaddition of azadienes with malononitrile using bifunctional squaramide as catalyst has been realized by our group [20] (Scheme 1a). Although considerable

\footnotetext{
* Corresponding author. Tel/Fax: +86-411-84379220; E-mail: ygzhou@dicp.ac.cn

This work was supported by the National Natural Science Foundation of China (21532006), Dalian Bureau of Science and Technology (2016RD07), and the Strategic Priority Program of the Chinese Academy of Sciences (XDB17020300).

DOI: S1872-2067(19)63396-6 | http://www.sciencedirect.com/science/journal/18722067 | Chin. J. Catal., Vol. 40, No. 10, October 2019
} 
a) Previous Works on Organocatalytic Systems

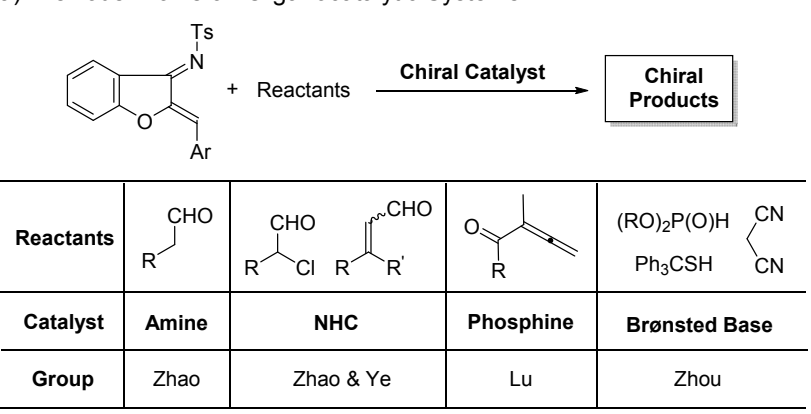

b) This Work: Chiral Brønsted Acid Catalysis

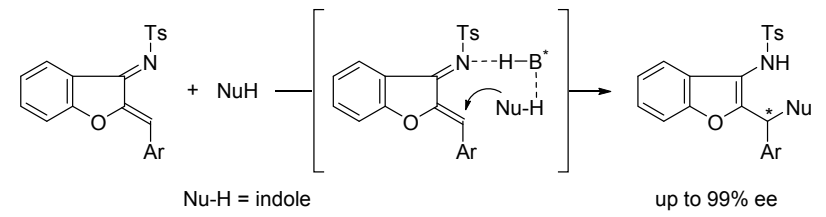

Scheme 1. Organocatalytic asymmetric reactions of azadienes.

progress has been achieved in asymmetric reactions of azadienes, the organocatalytic systems are still limited. Therefore, developing a new strategy for the asymmetric reactions of azadienes is still highly desirable.

Chiral Brønsted acids have been proved to be highly efficient and versatile catalysts for asymmetric transformations over the past decade [21-32]. Among these significant advances, chiral phosphoric acid-catalyzed Friedel-Crafts alkylation of electron-rich arenes is a facile and efficient approach for the construction of chiral hetero-triarylmethanes, which are ubiquitous and prevalent structural scaffolds in natural products, biologically active molecules and synthetic materials [33-58]. As our continuing efforts to the utilization of azadienes, we previously focused on chiral Brønsted base-catalyzed reactions of azadienes $[17,18,20]$. Considering that chiral Brønsted acids have been widely used to activate substrates through bifunctional catalysis mode, we envisioned that chiral Brønsted acid-catalyzed conjugate addition of indoles to azadienes could enable a facile approach to optically active hetero-triarylmethanes (Scheme 1b). To the best of our knowledge, chiral Brønsted acid catalysis has not been employed to the asymmetric reactions of azadienes. Herein, we reported bifunctional Brønsted acid-catalyzed conjugate addition of indoles to azadienes for the synthesis of chiral hetero-triarylmethanes with excellent enantioselectivities and broad substrate scope.

\section{Experimental}

\subsection{General methods}

Commercially available reagents and solvents were used without further purification. ${ }^{1} \mathrm{H}$ NMR and ${ }^{13} \mathrm{C}$ NMR spectra were recorded at room temperature in $\mathrm{CDCl}_{3}$ or DMSO-d6 on a 400 $\mathrm{MHz}$ instrument with tetramethylsilane (TMS) as internal standard. Flash column chromatography was performed on silica gel (200-300 mesh). All reactions were monitored by TLC analysis. Azadienes $\mathbf{1}$ could be conveniently synthesized from enones and sulfonamides according to the known literature procedures [13,16-18].

\subsection{General procedure for catalytic enantioselective conjugate addition}

To a solution of azadienes $1(0.20 \mathrm{mmol})$ and chiral phosphoric acid (R)-TRIP (A1) (7.5 mg, $0.01 \mathrm{mmol})$ in mesitylene $(3.0 \mathrm{~mL})$ at $-20^{\circ} \mathrm{C}$, indoles $2(0.20 \mathrm{mmol})$ was added. The reaction was stirred at $-20^{\circ} \mathrm{C}$ for $2-3 \mathrm{~d}$, which was monitored by thin-layer chromatography. The crude product was directly purified by silica gel column chromatography (eluent: hexanes/ethyl acetate $=10: 1$ to $5: 1$ ) to give the chiral hetero-triarylmethanes $\mathbf{3}$.

(+)- $N$-(2-((1H-Indol-3-yl) (phenyl)methyl)benzofuran-3-yl)4-methylbenzenesulfonamide (3aa): $95 \mathrm{mg}$, 96\% yield, yellow solid, m.p. $=106-108{ }^{\circ} \mathrm{C}$, new compound, $R_{\mathrm{f}}=0.25$ (hexanes/ethyl acetate 5:1), 89\% ee, $[\alpha]^{20} \mathrm{D}=+93.36(c 0.98$, EtOAc). ${ }^{1} \mathrm{H}$ NMR (400 MHz, DMSO- $\left.d_{6}\right) \delta 10.90(\mathrm{~s}, 1 \mathrm{H}), 10.02$ (s, 1H), 7.52 (d, $J=7.9 \mathrm{~Hz}, 2 \mathrm{H}$ ), 7.45 (d, $J=8.2 \mathrm{~Hz}, 1 \mathrm{H}$ ), 7.35 (d, $J=$ $8.1 \mathrm{~Hz}, 1 \mathrm{H}), 7.30-7.08(\mathrm{~m}, 9 \mathrm{H}), 7.07-6.95(\mathrm{~m}, 3 \mathrm{H}), 6.93-6.81$ $(\mathrm{m}, 2 \mathrm{H}), 5.80(\mathrm{~s}, 1 \mathrm{H}), 2.12(\mathrm{~s}, 3 \mathrm{H}) ;{ }^{13} \mathrm{C} \mathrm{NMR}(100 \mathrm{MHz}$, DMSO-d6) $\delta$ 154.8, 152.6, 143.0, 140.9, 136.9, 136.2, 129.3, 128.2, 128.1, 126.7, 126.4, 126.2, 125.9, 124.2, 123.7, 122.7, 121.0, 119.8, 118.8, 118.5, 114.0, 113.1, 111.5, 111.3, 38.5, 20.9. HPLC: Chiralcel IC column, $254 \mathrm{~nm}, 30^{\circ} \mathrm{C}, n$-hexane $/ i$-PrOH $=$ $90 / 10$, flow $=0.7 \mathrm{~mL} / \mathrm{min}$, retention time $21.2 \mathrm{~min}$ (major) and 23.9 min. HRMS calculated for $\mathrm{C}_{30} \mathrm{H}_{28} \mathrm{~N}_{3} \mathrm{O}_{3} \mathrm{~S}$ [M+NH$]^{+}$ 510.1846, found: 510.1849 .

(+)- $N$-(2-((2-Methyl-1H-indol-3-yl)(phenyl)methyl)benzofu ran-3-yl)-4-methylbenzenesulfonamide (3ab): $91 \mathrm{mg}$, 90\% yield, pale yellow solid, m.p. $=111-113{ }^{\circ} \mathrm{C}$, new compound, $R_{\mathrm{f}}=$ 0.30 (hexanes/ethyl acetate $=5: 1), 79 \%$ ee, $[\alpha]^{20} \mathrm{D}=+74.66(c$ 0.90, EtOAc). ${ }^{1} \mathrm{H}$ NMR (400 MHz, $\left.\mathrm{CDCl}_{3}\right) \delta 7.84(\mathrm{~s}, 1 \mathrm{H}), 7.63(\mathrm{~d}, J$ $=8.2 \mathrm{~Hz}, 2 \mathrm{H}), 7.33(\mathrm{~d}, J=8.2 \mathrm{~Hz}, 1 \mathrm{H}), 7.28(\mathrm{~d}, J=8.4 \mathrm{~Hz}, 1 \mathrm{H})$, 7.24-7.15 (m, 5H), 7.14-7.01 (m, 7H), 6.97-6.88 (m, 1H), 6.09 (s, 1H), 5.76 (s, 1H), 2.32 (s, 3H), $2.23(\mathrm{~s}, 3 \mathrm{H}) ;{ }^{13} \mathrm{C}$ NMR $(100$ $\mathrm{MHz}_{\mathrm{CDCl}}$ ) $\delta 156.5,153.1,144.1,140.1,136.6,135.2,132.9$, $129.7,128.4,128.3,127.9,127.6,126.6,126.1,124.3,123.1$, 121.3, 119.7, 119.3, 119.2, 113.4, 111.7, 110.4, 110.1, 38.7, 21.6, 12.4. HPLC: Chiralcel AD-H column, $254 \mathrm{~nm}, 30{ }^{\circ} \mathrm{C}$, $n$-hexane $/ i$-PrOH $=60 / 40$, flow $=0.8 \mathrm{~mL} / \mathrm{min}$, retention time $10.6 \mathrm{~min}$ (major) and $13.0 \mathrm{~min}$. HRMS calculated for $\mathrm{C}_{31} \mathrm{H}_{26} \mathrm{~N}_{2} \mathrm{NaO}_{3} \mathrm{~S}[\mathrm{M}+\mathrm{Na}]+529.1556$, found: 529.1555 .

(+)-Methyl-3-((3-((4-methylphenyl)sulfonamido)benzofura n-2-yl)(phenyl)methyl)-1H-indole-4-carboxylate (3ac): $94 \mathrm{mg}$, $85 \%$ yield, white solid, m.p. $=113-114{ }^{\circ} \mathrm{C}$, new compound, $R_{\mathrm{f}}=$ 0.10 (hexanes/ethyl acetate $=5: 1), 89 \%$ ee, $[\alpha]^{20} \mathrm{D}=+119.14(c$ 0.94, EtOAc). ${ }^{1} \mathrm{H}$ NMR (400 MHz, DMSO- $\left.d_{6}\right) \delta 11.30$ (d, $J=1.8$ $\mathrm{Hz}, 1 \mathrm{H}), 10.13$ (brs, 1H), 7.65 (d, J = 8.0 Hz, 1H), 7.59 (d, $J=8.1$ $\mathrm{Hz}, 2 \mathrm{H}), 7.43$ (d, $J=8.1 \mathrm{~Hz}, 1 \mathrm{H}), 7.34$ (d, $J=7.3 \mathrm{~Hz}, 1 \mathrm{H}), 7.29$ (d, $J$ $=7.6 \mathrm{~Hz}, 1 \mathrm{H}), 7.25-7.06(\mathrm{~m}, 6 \mathrm{H}), 7.01(\mathrm{~d}, J=7.0 \mathrm{~Hz}, 2 \mathrm{H}), 6.91(\mathrm{~d}$, $J=8.1 \mathrm{~Hz}, 2 \mathrm{H}), 6.78(\mathrm{~d}, J=2.0 \mathrm{~Hz}, 1 \mathrm{H}), 6.26(\mathrm{~s}, 1 \mathrm{H}), 3.56(\mathrm{~s}, 3 \mathrm{H})$, 1.96 (s, 3H); ${ }^{13} \mathrm{C}$ NMR (100 MHz, DMSO- $\left.d_{6}\right) \delta 168.0,156.4$, 152.6, 142.6, 141.5, 137.5, 137.0, 129.2, 128.5, 127.7, 126.8, $126.3,126.0,124.1,124.0,122.8,122.7,121.3,120.0,119.8$, 
115.7, 113.8, 112.8, 111.3, 51.6, 38.9, 20.7. HPLC: Chiralcel $\mathrm{AD}-\mathrm{H}$ column, $254 \mathrm{~nm}, 30^{\circ} \mathrm{C}, n$-hexane $/ i-\mathrm{PrOH}=60 / 40$, flow $=$ $0.8 \mathrm{~mL} / \mathrm{min}$, retention time $8.1 \mathrm{~min}$ and $24.6 \mathrm{~min}$ (major). HRMS calculated for $\mathrm{C}_{32} \mathrm{H}_{26} \mathrm{KN}_{2} \mathrm{O}_{5} \mathrm{~S}[\mathrm{M}+\mathrm{K}]+589.1194$, found: 589.1192.

(+)- $N$-(2-((5-Chloro-1H-indol-3-yl)(phenyl)methyl)benzofu ran-3-yl)-4-methylbenzenesulfonamide (3ad): $98 \mathrm{mg}$, 93\% yield, white solid, m.p. $=109-110{ }^{\circ} \mathrm{C}$, new compound, $R_{\mathrm{f}}=0.35$ (hexanes/ethyl acetate $=5: 1), 89 \%$ ee, $[\alpha]^{20} \mathrm{D}=+88.97(c$ 0.98, EtOAc). ${ }^{1} \mathrm{H}$ NMR (400 MHz, DMSO- $\left.d_{6}\right) \delta 11.15(\mathrm{~s}, 1 \mathrm{H}), 10.05(\mathrm{~s}$, 1H), 7.52 (d, $J=8.1 \mathrm{~Hz}, 2 \mathrm{H}), 7.45$ (d, $J=8.2 \mathrm{~Hz}, 1 \mathrm{H}), 7.39$ (d, $J=$ $8.6 \mathrm{~Hz}, 1 \mathrm{H}$ ), 7.30 (d, J = 7.6 Hz, 1H), 7.27-7.10 (m, 8H), 7.07 (dd, $J=8.6,1.9 \mathrm{~Hz}, 1 \mathrm{H}), 7.02-6.95(\mathrm{~m}, 3 \mathrm{H}), 5.77(\mathrm{~s}, 1 \mathrm{H}), 2.11(\mathrm{~s}, 3 \mathrm{H})$; ${ }^{13} \mathrm{C}$ NMR $\left(100 \mathrm{MHz}, \mathrm{DMSO}-d_{6}\right) \delta 154.2,152.6,143.0,140.6$, 136.8, 134.7, 129.3, 128.2, 128.1, 127.2, 126.7, 126.6, 125.8, $125.6,124.3,123.1,122.8,121.0,119.9,117.9,113.9,113.3$, 113.2, 111.3, 38.3, 20.8. HPLC: Chiralcel AD-H column, $254 \mathrm{~nm}$, $30{ }^{\circ} \mathrm{C}, n$-hexane $/ i$-PrOH $=60 / 40$, flow $=0.8 \mathrm{~mL} / \mathrm{min}$, retention time $9.9 \mathrm{~min}$ (major) and $12.1 \mathrm{~min}$. HRMS calculated for $\mathrm{C}_{30} \mathrm{H}_{27} \mathrm{ClN}_{3} \mathrm{O}_{3} \mathrm{~S}\left[\mathrm{M}+\mathrm{NH}_{4}\right]+544.1456$, found: 544.1457.

(+)- $N$-(2-((5-Methoxy-1H-indol-3-yl)(phenyl)methyl)benzof uran-3-yl)-4-methylbenzenesulfonamide (3ae): $96 \mathrm{mg}$, 92\% yield, white solid, m.p. $=105-106^{\circ} \mathrm{C}$, new compound, $R_{\mathrm{f}}=0.20$ (hexanes/ethyl acetate $=5: 1), 92 \%$ ee, $[\alpha]^{20} \mathrm{D}=+89.51(c$ 1.04, EtOAc). ${ }^{1} \mathrm{H}$ NMR $\left(400 \mathrm{MHz}, \mathrm{CDCl}_{3}\right) \delta 8.00(\mathrm{~s}, 1 \mathrm{H}), 7.59$ (d, $J=8.2$ $\mathrm{Hz}, 2 \mathrm{H}), 7.34(\mathrm{~d}, J=8.2 \mathrm{~Hz}, 1 \mathrm{H}), 7.25-7.15(\mathrm{~m}, 8 \mathrm{H}), 7.10(\mathrm{t}, J=$ $7.4 \mathrm{~Hz}, 1 \mathrm{H}), 6.97(\mathrm{~d}, J=8.1 \mathrm{~Hz}, 2 \mathrm{H}), 6.81(\mathrm{dd}, J=8.8,2.3 \mathrm{~Hz}, 1 \mathrm{H})$, $6.77(\mathrm{~d}, J=1.9 \mathrm{~Hz}, 1 \mathrm{H}), 6.73(\mathrm{~d}, J=1.9 \mathrm{~Hz}, 1 \mathrm{H}), 6.53-6.43(\mathrm{~m}$, $1 \mathrm{H}), 5.69(\mathrm{~s}, 1 \mathrm{H}), 3.69$ (s, 3H), $2.21(\mathrm{~s}, 3 \mathrm{H}) ;{ }^{13} \mathrm{C}$ NMR $(100 \mathrm{MHz}$, $\left.\mathrm{CDCl}_{3}\right) \delta 155.8,154.0,153.3,144.1,140.2,136.4,131.5,129.6$, $128.6,128.4,127.5,127.0,126.9,126.1,124.4,123.2,119.6$, 114.9, 113.1, 112.4, 112.0, 111.6, 101.5, 55.9, 39.8, 21.5. HPLC: Chiralcel AD-H column, $254 \mathrm{~nm}, 30{ }^{\circ} \mathrm{C}, n$-hexane $/ i$-PrOH $=$ $60 / 40$, flow $=0.8 \mathrm{~mL} / \mathrm{min}$, retention time $13.4 \mathrm{~min}$ (major) and 20.2 min. HRMS calculated for $\mathrm{C}_{31} \mathrm{H}_{25} \mathrm{~N}_{2} \mathrm{O}_{4} \mathrm{~S}[\mathrm{M}-\mathrm{H}]^{-} 521.1540$, found: 521.1565 .

(+)- $N$-(2-((5-(Benzyloxy)-1H-indol-3-yl)(phenyl)methyl)be nzofuran-3-yl)-4-methylbenzenesulfonamide (3af): $105 \mathrm{mg}$, 88\% yield, pale yellow solid, m.p. $=100-101{ }^{\circ} \mathrm{C}$, new compound, $R_{\mathrm{f}}=0.45$ (hexanes/ethyl acetate $=5: 1$ ), $88 \%$ ee, $[\alpha]^{20} \mathrm{D}=+77.74$ (c 0.40, EtOAc). ${ }^{1} \mathrm{H}$ NMR $\left(400 \mathrm{MHz}, \mathrm{CDCl}_{3}\right) \delta$ $7.98(\mathrm{~d}, J=1.8 \mathrm{~Hz}, 1 \mathrm{H}), 7.58(\mathrm{~d}, J=8.3 \mathrm{~Hz}, 2 \mathrm{H}), 7.41-7.29(\mathrm{~m}$, $6 \mathrm{H}), 7.25-7.16(\mathrm{~m}, 8 \mathrm{H}), 7.13-7.08(\mathrm{~m}, 1 \mathrm{H}), 6.94(\mathrm{~d}, J=8.1 \mathrm{~Hz}$, $2 \mathrm{H}), 6.89(\mathrm{dd}, J=8.8,2.4 \mathrm{~Hz}, 1 \mathrm{H}), 6.79(\mathrm{~d}, J=2.3 \mathrm{~Hz}, 1 \mathrm{H}), 6.74$ (d, $J=2.1 \mathrm{~Hz}, 1 \mathrm{H}), 6.35$ (s, 1H), $5.65(\mathrm{~s}, 1 \mathrm{H}), 4.94(\mathrm{~s}, 2 \mathrm{H}), 2.19$ (s, $3 \mathrm{H}) ;{ }^{13} \mathrm{C} \mathrm{NMR}\left(100 \mathrm{MHz}, \mathrm{CDCl}_{3}\right) \delta 155.8,153.3,153.1,144.0$, 140.1, 137. 7, 136.3, 131.6, 129.5, 128.6, 128.5, 128.4, 127.8, $127.6,127.5,126.9,126.8,126.0,124.3,124.3,123.1,119.5$, 115.0, 113.2, 113.0, 112.0, 111.6, 102.9, 70.8, 39.7, 21.4. HPLC: Chiralcel AD-H column, $254 \mathrm{~nm}, 30^{\circ} \mathrm{C}, n$-hexane $/ \mathrm{i}$-PrOH $=$ $60 / 40$, flow $=0.8 \mathrm{~mL} / \mathrm{min}$, retention time $18.1 \mathrm{~min}$ (major) and 22.2 min. HRMS calculated for $\mathrm{C}_{37} \mathrm{H}_{34} \mathrm{~N}_{3} \mathrm{O}_{4} \mathrm{~S}\left[\mathrm{M}+\mathrm{NH}_{4}\right]^{+}$ 616.2265, found: 616.2269 .

(+)-4-Methyl- $N$-(2-((6-methyl-1H-indol-3-yl)(phenyl)methy l)benzofuran-3-yl)benzenesulfonamide (3ag): $91 \mathrm{mg}, 90 \%$ yield, white solid, m.p. $=231-233^{\circ} \mathrm{C}$, new compound, $R_{\mathrm{f}}=0.10$ (hexanes/ethyl acetate $=5: 1), 93 \%$ ee, $[\alpha]^{20} \mathrm{D}=+103.80(c$ 0.92,
EtOAc). ${ }^{1} \mathrm{H}$ NMR $\left(400 \mathrm{MHz}, \mathrm{CDCl}_{3}\right) \delta 7.88(\mathrm{~s}, 1 \mathrm{H}), 7.58(\mathrm{~d}, J=8.3$ $\mathrm{Hz}, 2 \mathrm{H}), 7.34-7.28(\mathrm{~m}, 2 \mathrm{H}), 7.23-7.16(\mathrm{~m}, 4 \mathrm{H}), 7.14-7.05(\mathrm{~m}$, $5 \mathrm{H}), 7.02(\mathrm{~d}, J=8.1 \mathrm{~Hz}, 2 \mathrm{H}), 6.82(\mathrm{~d}, J=7.3 \mathrm{~Hz}, 1 \mathrm{H}), 6.72(\mathrm{~d}, J=$ $1.8 \mathrm{~Hz}, 1 \mathrm{H}), 6.05(\mathrm{~s}, 1 \mathrm{H}), 5.59(\mathrm{~s}, 1 \mathrm{H}), 2.41(\mathrm{~s}, 3 \mathrm{H}), 2.22(\mathrm{~s}, 3 \mathrm{H})$; ${ }^{13} \mathrm{C}$ NMR $\left(100 \mathrm{MHz}, \mathrm{CDCl}_{3}\right) \delta 155.6,153.4,144.1,140.2,136.9$, 136.4, 132.4, 129.7, 128.6, 128.5, 127.7, 126.9, 126.2, 124.4, 123.2, 122.9, 121.7, 119.7, 119.2, 115.2, 113.2, 111.7, 111.3, 39.9, 21.8, 21.6. HPLC: Chiralcel AD-H column, $254 \mathrm{~nm}, 30{ }^{\circ} \mathrm{C}$, $n$-hexane $/ i$-PrOH $=60 / 40$, flow $=0.8 \mathrm{~mL} / \mathrm{min}$, retention time $19.3 \mathrm{~min}$ and $21.2 \mathrm{~min}$ (major). HRMS calculated for $\mathrm{C}_{31} \mathrm{H}_{26} \mathrm{KN}_{2} \mathrm{O}_{3} \mathrm{~S}[\mathrm{M}+\mathrm{K}]+542.1296$, found: 542.1293.

(+)-4-Methyl- $N$-(2-((7-methyl- $1 H$-indol-3-yl)(phenyl)methy l)benzofuran-3-yl)benzenesulfonamide (3ah): $93 \mathrm{mg}$, 92\% yield, white solid, m.p. $=190-192{ }^{\circ} \mathrm{C}$, new compound, $R_{\mathrm{f}}=0.30$ (hexanes/ethyl acetate $=5: 1), 98 \%$ ee, $[\alpha]^{20} \mathrm{D}=+116.77(c 0.93$, EtOAc). ${ }^{1} \mathrm{H}$ NMR (400 MHz, $\left.\mathrm{CDCl}_{3}\right) \delta 7.97(\mathrm{~s}, 1 \mathrm{H}), 7.62(\mathrm{~d}, J=8.1$ $\mathrm{Hz}, 2 \mathrm{H}), 7.35$ (d, $J=8.4 \mathrm{~Hz}, 2 \mathrm{H}), 7.24-7.07$ (m, 8H), 7.05-6.90 $(\mathrm{m}, 4 \mathrm{H}), 6.79(\mathrm{~d}, J=1.6 \mathrm{~Hz}, 1 \mathrm{H}), 6.55-6.36(\mathrm{~m}, 1 \mathrm{H}), 5.68(\mathrm{~s}, 1 \mathrm{H})$, $2.43(\mathrm{~s}, 3 \mathrm{H}), 2.21(\mathrm{~s}, 3 \mathrm{H}) ;{ }^{13} \mathrm{C}$ NMR $\left(100 \mathrm{MHz}, \mathrm{CDCl}_{3}\right) \delta 155.7$, 153.3, 144.0, 140.2, 136.3, 135.9, 129.6, 128.6, 128.4, 127.6, 126.9, 126.2, 126.1, 124.4, 123.3, 123.2, 122.9, 120.5, 120.0, 119.7, 117.3, 115.7, 113.0, 111.6, 39.9, 21.5, 16.6. HPLC: Chiralcel AD-H column, $254 \mathrm{~nm}, 30{ }^{\circ} \mathrm{C}, n$-hexane $/ i$-PrOH $=60 / 40$, flow $=0.8 \mathrm{~mL} / \mathrm{min}$, retention time $12.8 \mathrm{~min}$ and $15.6 \mathrm{~min}$ (major). HRMS calculated for $\mathrm{C}_{31} \mathrm{H}_{25} \mathrm{~N}_{2} \mathrm{O}_{3} \mathrm{~S}[\mathrm{M}-\mathrm{H}]^{-}$505.1591, found: 505.1604.

(-)- $N$-(2-((1H-Indol-3-yl)(o-tolyl)methyl)benzofuran-3-yl)4-methylbenzenesulfonamide (3ba): $94 \mathrm{mg}$, 93\% yield, white solid, m.p. $=95-97^{\circ} \mathrm{C}$, new compound, $R_{\mathrm{f}}=0.25$ (hexanes/ethyl acetate $=5: 1), 93 \%$ ee, $[\alpha]^{20} \mathrm{D}=-54.68\left(c 0.94\right.$, EtOAc). ${ }^{1} \mathrm{H}$ NMR (400 MHz, $\left.\mathrm{CDCl}_{3}\right) \delta 8.01(\mathrm{~s}, 1 \mathrm{H}), 7.48(\mathrm{~d}, J=8.2 \mathrm{~Hz}, 2 \mathrm{H}), 7.36(\mathrm{~d}$, $J=7.5 \mathrm{~Hz}, 1 \mathrm{H}), 7.25(\mathrm{~d}, J=8.1 \mathrm{~Hz}, 2 \mathrm{H}), 7.17-7.04(\mathrm{~m}, 6 \mathrm{H})$, 6.99-6.89 (m, 5H), $6.49(\mathrm{~d}, J=1.4 \mathrm{~Hz}, 1 \mathrm{H}), 6.00(\mathrm{~s}, 1 \mathrm{H}), 5.74(\mathrm{~s}$, 1H), $2.17(\mathrm{~s}, 3 \mathrm{H}), 2.14(\mathrm{~s}, 3 \mathrm{H}) ;{ }^{13} \mathrm{C}$ NMR $\left(100 \mathrm{MHz}, \mathrm{CDCl}_{3}\right) \delta$ $154.5,153.3,144.0,138.5,136.5,136.4,136.1,130.6,129.6$, $128.5,127.5,127.1,126.5,126.2,124.5,124.0,123.2,122.5$, 119.9, 119.9, 119.1, 114.8, 113.7, 111.6, 111.5, 37.1, 21.6, 19.6. HPLC: Chiralcel AD-H column, $254 \mathrm{~nm}, 30^{\circ} \mathrm{C}, n$-hexane/i-PrOH $=60 / 40$, flow $=0.8 \mathrm{~mL} / \mathrm{min}$, retention time $12.2 \mathrm{~min}$ and 16.6 min (major). HRMS calculated for $\mathrm{C}_{31} \mathrm{H}_{26} \mathrm{KN}_{2} \mathrm{O}_{3} \mathrm{~S} \quad[\mathrm{M}+\mathrm{K}]^{+}$ 545.1296, found: 545.1291.

(+)- $N$-(2-((1H-Indol-3-yl) ( $m$-tolyl)methyl)benzofuran-3-yl)4-methylbenzenesulfonamide (3ca): $89 \mathrm{mg}$, 88\% yield, white solid, m.p. $=236-238{ }^{\circ} \mathrm{C}$, new compound, $R_{\mathrm{f}}=0.30$ (hexanes/ethyl acetate $=5: 1), 86 \%$ ee, $[\alpha]^{20} \mathrm{D}=+107.49(c 0.40$, EtOAc). ${ }^{1} \mathrm{H}$ NMR $\left(400 \mathrm{MHz}, \mathrm{CDCl}_{3}\right) \delta 8.05(\mathrm{~s}, 1 \mathrm{H}), 7.62(\mathrm{~d}, J=8.2$ $\mathrm{Hz}, 2 \mathrm{H}), 7.42-7.29(\mathrm{~m}, 3 \mathrm{H}), 7.25-7.07$ (m, 5H), 7.05-6.92 (m, $6 \mathrm{H}), 6.79(\mathrm{~d}, J=1.7 \mathrm{~Hz}, 1 \mathrm{H}), 6.37(\mathrm{~s}, 1 \mathrm{H}), 5.61(\mathrm{~s}, 1 \mathrm{H}), 2.26(\mathrm{~s}$, 3H), 2.19 (s, 3H); ${ }^{13} \mathrm{C}$ NMR $\left(100 \mathrm{MHz}, \mathrm{CDCl}_{3}\right) \delta 155.8,153.4$, 144.1, 140.1, 138.0, 136.4, 129.6, 129.3, 128.3, 127.8, 127.6, 126.6, 126.3, 125.6, 124.4, 123.6, 123.2, 122.3, 119.8, 119.7, $119.5,115.3,113.0,111.7,111.3,39.8,21.6,21.5$. HPLC: Chiralcel AD-H column, $254 \mathrm{~nm}, 30{ }^{\circ} \mathrm{C}, n$-hexane $/ i$-PrOH $=60 / 40$, flow $=0.8 \mathrm{~mL} / \mathrm{min}$, retention time $11.7 \mathrm{~min}$ and $22.3 \mathrm{~min}$ (major). HRMS calculated for $\mathrm{C}_{31} \mathrm{H}_{26} \mathrm{KN}_{2} \mathrm{O}_{3} \mathrm{~S}[\mathrm{M}+\mathrm{K}]^{+}$545.1296, found: 545.1300 .

(+)- $N$-(2-((1H-Indol-3-yl)( $p$-tolyl)methyl)benzofuran-3-yl)- 
4-methylbenzenesulfonamide (3da): $91 \mathrm{mg}, 90 \%$ yield, pale yellow solid, m.p. $=103-104{ }^{\circ} \mathrm{C}$, new compound, $R_{\mathrm{f}}=0.30$ (hexane s/ethyl acetate $=5: 1), 89 \%$ ee, $[\alpha]^{20_{D}}=+17.50(c 0.40$, EtOAc). ${ }^{1} \mathrm{H}$ NMR $\left(400 \mathrm{MHz}, \mathrm{CDCl}_{3}\right) \delta 8.02(\mathrm{~s}, 1 \mathrm{H}), 7.60(\mathrm{~d}, J=8.3$ $\mathrm{Hz}, 2 \mathrm{H}), 7.37-7.28(\mathrm{~m}, 3 \mathrm{H}), 7.24-7.17(\mathrm{~m}, 2 \mathrm{H}), 7.14(\mathrm{~m}, 2 \mathrm{H})$, $7.05-6.97(\mathrm{~m}, 7 \mathrm{H}), 6.79(\mathrm{~d}, J=1.8 \mathrm{~Hz}, 1 \mathrm{H}), 6.24(\mathrm{~s}, 1 \mathrm{H}), 5.60(\mathrm{~s}$, 1H), 2.30 (s, 3H), 2.21 (s, 3H); ${ }^{13} \mathrm{C}$ NMR $\left(100 \mathrm{MHz} \mathrm{CDCl}_{3}\right) \delta$ $155.9,153.4,144.1,137.2,136.5,136.4,136.4,129.7,129.2$, $128.5,127.6,126.6,126.2,124.4,123.5,123.2,122.4,119.8$, 119.7, 119.6, 115.5, 113.0, 111.6, 111.3, 39.5, 21.5, 21.2. HPLC: Chiralcel AD-H column, $254 \mathrm{~nm}, 30{ }^{\circ} \mathrm{C}, n$-hexane $/ i$-PrOH $=$ $60 / 40$, flow $=0.8 \mathrm{~mL} / \mathrm{min}$, retention time $11.9 \mathrm{~min}$ and 23.4 min (major). HRMS calculated for $\mathrm{C}_{31} \mathrm{H}_{26} \mathrm{KN}_{2} \mathrm{O}_{3} \mathrm{~S}[\mathrm{M}+\mathrm{K}]^{+}$ 545.1296, found: 545.1292 .

(+)- $N$-(2-((1H-Indol-3-yl)(4-isopropylphenyl)methyl)benzof uran-3-yl)-4-methylbenzenesulfonamidee (3ea): $95 \mathrm{mg}, 89 \%$ yield, white solid, m.p. $=110-112{ }^{\circ} \mathrm{C}$, new compound, $R_{\mathrm{f}}=0.45$ (hexanes/ethyl acetate $=10: 1), 90 \%$ ee, $[\alpha]^{20} \mathrm{D}=+142.99(c$ 0.40 , EtOAc). ${ }^{1} \mathrm{H}$ NMR (400 MHz, DMSO- $\left.d_{6}\right) \delta 10.90$ (d, $J=1.7$ $\mathrm{Hz}, 1 \mathrm{H}), 10.01(\mathrm{~s}, 1 \mathrm{H}), 7.55$ (d, $J=8.2 \mathrm{~Hz}, 2 \mathrm{H}), 7.45$ (d, $J=8.2 \mathrm{~Hz}$, $1 \mathrm{H}), 7.37(\mathrm{~d}, J=8.1 \mathrm{~Hz}, 1 \mathrm{H}), 7.27-7.17(\mathrm{~m}, 3 \mathrm{H}), 7.15-7.03(\mathrm{~m}$, $6 \mathrm{H}), 7.00$ (d, $J=8.2 \mathrm{~Hz}, 2 \mathrm{H}), 6.95-6.86(\mathrm{~m}, 2 \mathrm{H}), 5.79(\mathrm{~s}, 1 \mathrm{H})$, 2.98-2.71 (m, 1H), 2.12 (s, 3H), 1.51-0.70 (m, 6H); ${ }^{13} \mathrm{C}$ NMR (100 MHz, DMSO-d6) $\delta 155.1,152.6,146.4,143.0,138.3,137.0$, $136.2,129.3,128.2,126.7,126.3,126.0,125.9,124.1,123.5$, $122.7,121.0,119.7,118.8,118.5,114.2,112.9,111.5,111.3$, 38.1, 33.0, 23.9, 23.8, 20.9. HPLC: Chiralcel AD-H column, 254 $\mathrm{nm}, 30{ }^{\circ} \mathrm{C}, n$-hexane $/ i$-PrOH $=60 / 40$, flow $=0.8 \mathrm{~mL} / \mathrm{min}$, retention time $11.9 \mathrm{~min}$ and $20.3 \mathrm{~min}$ (major). HRMS calculated for $\mathrm{C}_{33} \mathrm{H}_{30} \mathrm{KN}_{2} \mathrm{O}_{3} \mathrm{~S}[\mathrm{M}+\mathrm{K}]+573.1609$, found: 573.1610.

(-)- $N$-(2-((1H-Indol-3-yl)(naphthalen-1-yl)methyl)benzofur an-3-yl)-4-methylbenzenesulfonamide (3fa): $97 \mathrm{mg}$, 89\% yield, pale yellow solid, m.p. $=240-241^{\circ} \mathrm{C}$, new compound, $R_{\mathrm{f}}=0.30$ (hexanes/ethyl acetate $=4: 1), 96 \%$ ee, $[\alpha]^{20} \mathrm{D}=-88.39(c 0.50$, EtOAc). ${ }^{1} \mathrm{H}$ NMR (400 MHz, DMSO- $\left.d_{6}\right) \delta 10.90(\mathrm{~s}, 1 \mathrm{H}), 10.15(\mathrm{~s}$, $1 \mathrm{H}), 8.18-8.09(\mathrm{~m}, 1 \mathrm{H}), 7.98-7.90(\mathrm{~m}, 1 \mathrm{H}), 7.81(\mathrm{~d}, J=8.1 \mathrm{~Hz}$, 1H), 7.53-7.30 (m, 9H), $7.26(\mathrm{~d}, J=7.1 \mathrm{~Hz}, 1 \mathrm{H}), 7.22-7.09(\mathrm{~m}$, $2 \mathrm{H}), 7.05(\mathrm{t}, J=7.5 \mathrm{~Hz}, 1 \mathrm{H}), 6.88(\mathrm{t}, J=7.4 \mathrm{~Hz}, 1 \mathrm{H}), 6.81-6.64$ $(\mathrm{m}, 4 \mathrm{H}), 2.05(\mathrm{~s}, 3 \mathrm{H}) ;{ }^{13} \mathrm{C}$ NMR (100 MHz, DMSO- $\left.d_{6}\right) \delta 154.7$, 152.7, 142.8, 137.2, 137.0, 136.3, 133.5, 131.0, 129.1, 128.7, $127.2,126.5,126.3,126.1,126.0,125.5,125.4,124.5,124.2$, 123.8, 122.8, 121.2, 119.8, 118.7, 118.6, 114.5, 113.5, 111.6, 111.3, 35.2, 20.9. HPLC: Chiralcel AD-H column, $254 \mathrm{~nm}, 30^{\circ} \mathrm{C}$, $n$-hexane $/ i-\mathrm{PrOH}=60 / 40$, flow $=0.8 \mathrm{~mL} / \mathrm{min}$, retention time $14.5 \mathrm{~min}$ and $22.9 \mathrm{~min}$ (major). HRMS calculated for $\mathrm{C}_{34} \mathrm{H}_{26} \mathrm{KN}_{2} \mathrm{O}_{3} \mathrm{~S}[\mathrm{M}+\mathrm{K}]+581.1296$, found:581.1301.

(+)-N-(2-((1H-Indol-3-yl)(4-methoxyphenyl)methyl)benzof uran-3-yl)-4-methylbenzenesulfonamidee (3ga): $99 \mathrm{mg}, 95 \%$ yield, tan solid, m.p. $=94-96{ }^{\circ} \mathrm{C}$, new compound, $R_{\mathrm{f}}=0.20$ (hexanes/ethyl acetate $=5: 1), 76 \%$ ee, $[\alpha]^{20} \mathrm{D}=+125.16(c 0.60$, EtOAc). ${ }^{1} \mathrm{H}$ NMR $\left(400 \mathrm{MHz}, \mathrm{CDCl}_{3}\right) \delta 7.99(\mathrm{~s}, 1 \mathrm{H}), 7.57(\mathrm{~d}, J=8.3$ $\mathrm{Hz}, 2 \mathrm{H}), 7.33-7.25(\mathrm{~m}, 3 \mathrm{H}), 7.21-7.09(\mathrm{~m}, 4 \mathrm{H}), 7.08-7.02(\mathrm{~m}$, $2 \mathrm{H}), 7.00-6.95(\mathrm{~m}, 3 \mathrm{H}), 6.76(\mathrm{~s}, 1 \mathrm{H}), 6.74-6.69(\mathrm{~m}, 2 \mathrm{H})$, 6.23-6.10 (m, 1H) $5.57(\mathrm{~s}, 1 \mathrm{H}), 3.73(\mathrm{~s}, 3 \mathrm{H}), 2.19(\mathrm{~s}, 3 \mathrm{H}) ;{ }^{13} \mathrm{C}$ NMR $\left(100 \mathrm{MHz}, \mathrm{CDCl}_{3}\right) \delta 158.5,156.0,153.4,144.1,136.4$, $132.3,129.7,129.6,127.6,126.6,126.2,124.4,123.4,123.2$, $122.4,119.8,119.7,119.6,115.7,113.9,112.8,111.7,111.3$,
55.3, 39.1, 21.5. HPLC: Chiralcel IA column, $254 \mathrm{~nm}, 30{ }^{\circ} \mathrm{C}$, $n$-hexane $/ i$-PrOH $=60 / 40$, flow $=0.8 \mathrm{~mL} / \mathrm{min}$, retention time $13.5 \mathrm{~min}$ (major) and $16.6 \mathrm{~min}$. HRMS calculated for $\mathrm{C}_{31} \mathrm{H}_{26} \mathrm{KN}_{2} \mathrm{O}_{4} \mathrm{~S}[\mathrm{M}+\mathrm{K}]+561.1245$, found: 561.1250.

(+)- $N$-(2-((3-Bromophenyl)(1H-indol-3-yl)methyl)benzofur an-3-yl)-4-methylbenzenesulfonamide (3ha): $105 \mathrm{mg}, 92 \%$ yield, white solid, m.p. $=116-118{ }^{\circ} \mathrm{C}$, new compound, $R_{\mathrm{f}}=0.20$ (hexanes/ethyl acetate $=5: 1), 91 \%$ ee, $[\alpha]^{20} \mathrm{D}=+78.28(c 1.05$, EtOAc). ${ }^{1} \mathrm{H}$ NMR (400 MHz, $\left.\mathrm{CDCl}_{3}\right) \delta 8.06(\mathrm{~s}, 1 \mathrm{H}), 7.61(\mathrm{~d}, J=8.2$ $\mathrm{Hz}, 2 \mathrm{H}), 7.42-7.29(\mathrm{~m}, 4 \mathrm{H}), 7.26-7.18(\mathrm{~m}, 4 \mathrm{H}), 7.17-7.08(\mathrm{~m}$, $3 \mathrm{H}), 7.08-7.00(\mathrm{~m}, 3 \mathrm{H}), 6.88(\mathrm{~d}, J=1.6 \mathrm{~Hz}, 1 \mathrm{H}), 6.14(\mathrm{~s}, 1 \mathrm{H})$, $5.65(\mathrm{~s}, 1 \mathrm{H}), 2.23(\mathrm{~s}, 3 \mathrm{H}) ;{ }^{13} \mathrm{C} \mathrm{NMR}\left(100 \mathrm{MHz}, \mathrm{CDCl}_{3}\right) \delta 155.2$, 153.4, 144.2, 142.6, 136.4, 131.6, 130.1, 130.0, 129.7, 127.6, $127.4,126.4,126.1,124.7,123.6,123.4,122.6,122.5,120.0$, 119.6, 119.4, 114.8, 113.2, 111.8, 111.4, 39.3, 21.6. HPLC: Chiralcel AD-H column, $254 \mathrm{~nm}, 30{ }^{\circ} \mathrm{C}, n$-hexane $/ i$-PrOH $=60 / 40$, flow $=0.8 \mathrm{~mL} / \mathrm{min}$, retention time $12.0 \mathrm{~min}$ and $18.4 \mathrm{~min}$ (major). HRMS calculated for $\mathrm{C}_{30} \mathrm{H}_{22} \mathrm{BrN}_{2} \mathrm{O}_{3} \mathrm{~S}[\mathrm{M}-\mathrm{H}]^{-}$569.0540, found: 569.0541.

(+)- $N$-(2-((3-Chlorophenyl)(1H-indol-3-yl)methyl)benzofur an-3-yl)-4-methylbenzenesulfonamide (3ia): $95 \mathrm{mg}, 90 \%$ yield, pale yellow solid, m.p. $=234-236{ }^{\circ} \mathrm{C}$, new compound, $R_{\mathrm{f}}=0.35$ (hexanes/ethyl acetate $=4: 1), 92 \%$ ee, $[\alpha]^{20} \mathrm{D}=+139.12(c 0.80$, EtOAc). ${ }^{1} \mathrm{H}$ NMR (400 MHz, DMSO- $\left.d_{6}\right) \delta 10.98(\mathrm{~d}, J=1.7 \mathrm{~Hz}, 1 \mathrm{H})$, 10.12 (s, 1H), 7.52 (d, $J=8.2 \mathrm{~Hz}, 2 \mathrm{H}), 7.47$ (d, $J=8.1 \mathrm{~Hz}, 1 \mathrm{H}$ ), $7.37(\mathrm{t}, J=8.0 \mathrm{~Hz}, 2 \mathrm{H}), 7.28-7.13(\mathrm{~m}, 7 \mathrm{H}), 7.08(\mathrm{t}, J=7.3 \mathrm{~Hz}$, 1H), 6.96-6.88 (m, 4H), $5.80(\mathrm{~s}, 1 \mathrm{H}), 2.06(\mathrm{~s}, 3 \mathrm{H}) ;{ }^{13} \mathrm{C}$ NMR $(100$ MHz, DMSO-d $d_{6} \delta 153.7,152.8,143.5,143.1,136.7,136.3$, $132.9,130.1,129.4,127.9,127.0,126.7,126.6,126.0,125.8$, 124.5, 123.9, 123.0, 121.3, 120.1, 118.8, 118.7, 113.5, 113.4, 111.7, 111.5, 38.2, 20.9. HPLC: Chiralcel AD-H column, $254 \mathrm{~nm}$, $30{ }^{\circ} \mathrm{C}, n$-hexane $/ i-\mathrm{PrOH}=60 / 40$, flow $=0.8 \mathrm{~mL} / \mathrm{min}$, retention time $11.7 \mathrm{~min}$ and $17.0 \mathrm{~min}$ (major). HRMS calculated for $\mathrm{C}_{30} \mathrm{H}_{23} \mathrm{ClKN}_{2} \mathrm{O}_{3} \mathrm{~S}[\mathrm{M}+\mathrm{K}]+565.0749$, found: 565.0746.

(+)- $N$-(2-((4-Chlorophenyl)(1H-indol-3-yl)methyl)benzofur an-3-yl)-4-methylbenzenesulfonamide (3ja): $95 \mathrm{mg}, 90 \%$ yield, white solid, m.p. $=230-232{ }^{\circ} \mathrm{C}$, new compound, $R_{\mathrm{f}}=0.25$ (hexanes/ethyl acetate $=5: 1), 95 \%$ ee, $[\alpha]^{20} \mathrm{D}=+94.62(c 0.95$, EtOAc). ${ }^{1} \mathrm{H}$ NMR (400 MHz, $\left.\mathrm{CDCl}_{3}\right) \delta 8.07(\mathrm{~s}, 1 \mathrm{H}), 7.59$ (d, $J=8.2$ $\mathrm{Hz}, 2 \mathrm{H}), 7.33(\mathrm{t}, J=8.1 \mathrm{~Hz}, 2 \mathrm{H}), 7.25-7.08(\mathrm{~m}, 9 \mathrm{H}), 7.05-6.95$ $(\mathrm{m}, 3 \mathrm{H}), 6.83(\mathrm{~d}, J=1.7 \mathrm{~Hz}, 1 \mathrm{H}), 6.46(\mathrm{~s}, 1 \mathrm{H}), 5.70(\mathrm{~s}, 1 \mathrm{H}), 2.22$ (s, 3H); ${ }^{13} \mathrm{C}$ NMR $\left(100 \mathrm{MHz}, \mathrm{CDCl}_{3}\right) \delta 155.5,153.4,144.2,138.8$, $136.4,136.3,132.7,130.0,129.7,128.6,127.6,126.4,126.0$, 124.6, 123.6, 123.3, 122.5, 120.0, 119.6, 119.5, 115.0, 113.1, 111.7, 111.4, 39.1, 21.5. HPLC: Chiralcel AD-H column, $254 \mathrm{~nm}$, $30{ }^{\circ} \mathrm{C}, n$-hexane $/ i$-PrOH $=60 / 40$, flow $=0.8 \mathrm{~mL} / \mathrm{min}$, retention time $13.2 \mathrm{~min}$ and $22.8 \mathrm{~min}$ (major). HRMS calculated for $\mathrm{C}_{30} \mathrm{H}_{23} \mathrm{ClKN}_{2} \mathrm{O}_{3} \mathrm{~S}[\mathrm{M}+\mathrm{K}]+565.0749$, found: 565.0745.

(+)- $N$-(2-((1H-Indol-3-yl)(phenyl)methyl)-5-methylbenzofu ran-3-yl)-4-methylbenzenesulfonamide (3ka): $90 \mathrm{mg}, 89 \%$ yield, yellow solid, m.p. $=233-234^{\circ} \mathrm{C}$, new compound, $R_{\mathrm{f}}=0.35$ (hexanes/ethyl acetate $=5: 1), 84 \%$ ee, $[\alpha]^{20} \mathrm{D}=+86.44(c 0.45$, EtOAc). ${ }^{1} \mathrm{H}$ NMR (400 MHz, DMSO- $\left.d_{6}\right) \delta 10.91(\mathrm{~s}, 1 \mathrm{H}), 9.91(\mathrm{~s}$, 1H), $7.54(\mathrm{~d}, J=7.7 \mathrm{~Hz}, 2 \mathrm{H}), 7.36(\mathrm{~d}, J=7.9 \mathrm{~Hz}, 1 \mathrm{H}), 7.30(\mathrm{~d}, J=$ $8.3 \mathrm{~Hz}, 1 \mathrm{H}), 7.27-7.13(\mathrm{~m}, 6 \mathrm{H}), 7.12-6.94(\mathrm{~m}, 4 \mathrm{H}), 6.93-6.84$ (m, 2H), $6.81(\mathrm{~s}, 1 \mathrm{H}), 5.79(\mathrm{~s}, 1 \mathrm{H}), 2.24(\mathrm{~s}, 3 \mathrm{H}), 2.16(\mathrm{~s}, 3 \mathrm{H}) ;{ }^{13} \mathrm{C}$ NMR (100 MHz, DMSO-d6) $\delta$ 155.5, 151.2, 143.1, 141.1, 137.2, 
$136.4,131.7,129.4,128.3,128.1,126.9,126.5,126.3,126.0$, $125.2,123.8,121.1,119.5,118.9,118.6,114.2,112.9,111.6$, 110.9, 38.6, 20.9, 20.8. HPLC: Chiralcel IC column, $254 \mathrm{~nm}, 30$ ${ }^{\circ} \mathrm{C}, n$-hexane $/ i$-PrOH $=90 / 10$, flow $=0.7 \mathrm{~mL} / \mathrm{min}$, retention time $19.3 \mathrm{~min}$ (major) and $24.1 \mathrm{~min}$. HRMS calculated for $\mathrm{C}_{31} \mathrm{H}_{25} \mathrm{~N}_{2} \mathrm{O}_{3} \mathrm{~S}[\mathrm{M}-\mathrm{H}]^{-}$505.1591, found: 505.1608.

(+)- $N$-(2-((1H-Indol-3-yl)(phenyl)methyl)-6-methylbenzofu ran-3-yl)-4-methylbenzenesulfonamide (3la): $93 \mathrm{mg}, 90 \%$ yield, yellow solid, m.p. $=214-216{ }^{\circ} \mathrm{C}$, new compound, $R_{\mathrm{f}}=0.35$ (hexanes/ethyl acetate $=5: 1), 91 \%$ ee, $[\alpha]^{20} \mathrm{D}=+70.85(c 0.47$, EtOAc). ${ }^{1} \mathrm{H}$ NMR $\left(400 \mathrm{MHz}, \mathrm{CDCl}_{3}\right) \delta 8.02(\mathrm{~s}, 1 \mathrm{H}), 7.60(\mathrm{~d}, J=8.3$ $\mathrm{Hz}, 2 \mathrm{H}), 7.34$ (d, $J=8.2 \mathrm{~Hz}, 1 \mathrm{H}), 7.26-7.17(\mathrm{~m}, 5 \mathrm{H}), 7.17-7.11$ (m, 4H), 7.05-6.99 (m, 3H), 6.99-6.94 (m, 1H), $6.81(\mathrm{~d}, J=1.7$ $\mathrm{Hz}, 1 \mathrm{H}), 6.05$ (s, 1H), 5.60 (s, 1H), 2.40 (s, 3H), 2.22 (s, 3H); ${ }^{13} \mathrm{C}$ NMR $\left(100 \mathrm{MHz}, \mathrm{CDCl}_{3}\right) \delta 154.8,153.8,144.1,140.3,136.4$, $136.4,134.8,129.7,128.6,128.5,127.7,126.9,126.6,124.6$, $123.6,123.5,122.5,119.9,119.5,119.2,115.5,113.0,111.9$, 111.3, 39.9, 21.7, 21.6. HPLC: Chiralcel IC column, $254 \mathrm{~nm}, 30$ ${ }^{\circ} \mathrm{C}, n$-hexane $/ i$-PrOH $=90 / 10$, flow $=0.7 \mathrm{~mL} / \mathrm{min}$, retention time $22.1 \mathrm{~min}$ (major) and $26.7 \mathrm{~min}$. HRMS calculated for $\mathrm{C}_{31} \mathrm{H}_{25} \mathrm{~N}_{2} \mathrm{O}_{3} \mathrm{~S}$ [M-H] $]^{-}$505.1591, found: 505.1627.

(+)- $N$-(2-((1H-Indol-3-yl)(phenyl)methyl)benzofuran-3-yl)4-nitrobenzenesulfonamide (3ma): $92 \mathrm{mg}, 88 \%$ yield, pink solid, m.p. $=127-128{ }^{\circ} \mathrm{C}$, new compound, $R_{\mathrm{f}}=0.40$ (hexanes/ethyl acetate $=5: 1), 75 \%$ ee, $[\alpha]^{20} \mathrm{D}=+61.73(c \quad 0.46$, EtOAc). ${ }^{1} \mathrm{H}$ NMR $\left(400 \mathrm{MHz}, \mathrm{CDCl}_{3}\right) \delta 8.01(\mathrm{~s}, 1 \mathrm{H}), 7.83-7.69(\mathrm{~m}$, 4H), 7.52-7.39 (m, 2H), 7.35-7.26 (m, 3H), 7.24-7.18 (m, 3H), $7.20-7.09(\mathrm{~m}, 4 \mathrm{H}), 6.97(\mathrm{t}, J=7.5 \mathrm{~Hz}, 1 \mathrm{H}), 6.81(\mathrm{~d}, J=2.1 \mathrm{~Hz}$, 1H), 6.67-6.55 (m, 1H), 5.53 (s, 1H); ${ }^{13} \mathrm{C}$ NMR (100 MHz, $\left.\mathrm{CDCl}_{3}\right)$ $\delta 156.0,153.5,149.8,144.5,139.8,136.1,128.6,128.4,127.2$, $126.3,125.8,125.0,123.9,123.7,123.2,122.9,120.1,119.7$, 118.9, 115.2, 112.1, 111.9, 111.6, 39.6. HPLC: Chiralcel IC column, $254 \mathrm{~nm}, 30{ }^{\circ} \mathrm{C}, n$-hexane $/ i$-PrOH $=90 / 10$, flow $=0.7$ $\mathrm{mL} / \mathrm{min}$, retention time $18.5 \mathrm{~min}$ and $22.9 \mathrm{~min}$ (major). HRMS calculated for $\mathrm{C}_{29} \mathrm{H}_{25} \mathrm{~N}_{4} \mathrm{O}_{5} \mathrm{~S} \quad\left[\mathrm{M}+\mathrm{NH}_{4}\right]^{+}$541.1540, found: 541.1543.

(+)- $N$-(2-((1H-Indol-3-yl)(phenyl)methyl)benzofuran-3-yl) methanesulfonamide (3na): $72 \mathrm{mg}, 86 \%$ yield, pink solid, m.p. $=110-112{ }^{\circ} \mathrm{C}$, new compound, $R_{\mathrm{f}}=0.20$ (hexanes/ethyl acetate $=5: 1), 85 \%$ ee, $[\alpha]^{20} \mathrm{D}=+96.94\left(c\right.$ 0.72, EtOAc). ${ }^{1} \mathrm{H}$ NMR $(400$ $\left.\mathrm{MHz} \mathrm{CDCl}_{3}\right) \delta 8.09(\mathrm{~s}, 1 \mathrm{H}), 7.79-7.59(\mathrm{~m}, 1 \mathrm{H}), 7.48-7.37(\mathrm{~m}$, $4 \mathrm{H}), 7.36-7.26(\mathrm{~m}, 5 \mathrm{H}), 7.25-7.22(\mathrm{~m}, 1 \mathrm{H}), 7.17(\mathrm{t}, J=7.6 \mathrm{~Hz}$, $1 \mathrm{H}), 7.03(\mathrm{t}, J=7.5 \mathrm{~Hz}, 1 \mathrm{H}), 6.92(\mathrm{~d}, J=2.3 \mathrm{~Hz}, 1 \mathrm{H}), 6.24(\mathrm{~s}, 1 \mathrm{H})$, $6.11(\mathrm{~s}, 1 \mathrm{H}), 2.78(\mathrm{~s}, 3 \mathrm{H}) ;{ }^{13} \mathrm{C}$ NMR $\left(100 \mathrm{MHz}, \mathrm{CDCl}_{3}\right) \delta 155.9$, $153.5,140.2,136.5,128.7,127.2,126.6,126.0,124.8,123.8$, 123.6, 122.6, 120.0, 119.4, 119.3, 115.3, 113.1, 112.0, 111.5, 40.2, 40.1. HPLC: Chiralcel IC column, $254 \mathrm{~nm}, 30{ }^{\circ} \mathrm{C}$, $n$-hexane $/ i$-PrOH $=90 / 10$, flow $=0.7 \mathrm{~mL} / \mathrm{min}$, retention time $24.9 \mathrm{~min}$ (major) and $28.3 \mathrm{~min}$. HRMS calculated for $\mathrm{C}_{24} \mathrm{H}_{20} \mathrm{~N}_{2} \mathrm{NaO}_{3} \mathrm{~S}[\mathrm{M}+\mathrm{Na}]+439.1087$, found: 439.1085 .

(+)- $N$-(2-((1H-Indol-3-yl)(phenyl)methyl)benzofuran-3-yl) methanesulfonamide (3oa): $83 \mathrm{mg}, 80 \%$ yield, yellow solid, m.p. $=175-177{ }^{\circ} \mathrm{C}$, new compound, $R_{\mathrm{f}}=0.45$ (hexanes $/$ ethyl acetate $=5: 1), 83 \%$ ee, $[\alpha]^{20} \mathrm{D}=+84.74\left(c 0.40\right.$, EtOAc). ${ }^{1} \mathrm{H}$ NMR (400 MHz, DMSO- $\left.d_{6}\right) \delta 10.92(\mathrm{~d}, J=1.6 \mathrm{~Hz}, 1 \mathrm{H}), 9.93(\mathrm{~s}, 1 \mathrm{H})$, 7.49 (d, $J=8.1 \mathrm{~Hz}, 1 \mathrm{H}), 7.38$ (d, $J=8.1 \mathrm{~Hz}, 1 \mathrm{H}), 7.32$ (d, $J=7.6$ $\mathrm{Hz}, 1 \mathrm{H}), 7.27-7.12(\mathrm{~m}, 8 \mathrm{H}), 7.07(\mathrm{t}, J=7.3 \mathrm{~Hz}, 1 \mathrm{H}), 6.91(\mathrm{t}, J=$
$7.5 \mathrm{~Hz}, 1 \mathrm{H}), 6.86(\mathrm{~d}, J=2.1 \mathrm{~Hz}, 1 \mathrm{H}), 6.66(\mathrm{~s}, 2 \mathrm{H}), 5.67(\mathrm{~s}, 1 \mathrm{H})$, 2.39 (s, 6H), $2.00(\mathrm{~s}, 3 \mathrm{H}) ;{ }^{13} \mathrm{C}$ NMR $\left(100 \mathrm{MHz}, \mathrm{DMSO}-d_{6}\right) \delta 156.2$, 152.7, 141.8, 140.8, 138.5, 136.3, 134.3, 131.5, 128.2, 128.1, 126.5, 126.4, 126.2, 124.2, 123.5, 122.9, 121.1, 119.7, 118.7, 118.5, 113.9, 112.6, 111.5, 111.4, 38.7, 22.7, 20.3. HPLC: Chiralcel AD-H column, $254 \mathrm{~nm}, 30{ }^{\circ} \mathrm{C}, n$-hexane $/ i$-PrOH $=70 / 30$, flow $=0.8 \mathrm{~mL} / \mathrm{min}$, retention time $12.2 \mathrm{~min}$ and $18.1 \mathrm{~min}$ (major). HRMS calculated for $\mathrm{C}_{32} \mathrm{H}_{32} \mathrm{~N}_{3} \mathrm{O}_{3} \mathrm{~S}\left[\mathrm{M}+\mathrm{NH}_{4}\right]^{+} 538.2159$, found: 538.2156.

(S)-(-)- $N$-(2-((1H-Indol-3-yl)(phenyl)methyl)benzofuran-3yl)-4-methylbenzenesulfonamide (3fh): $104 \mathrm{mg}$, 93\% yield, pink solid, m.p. $=145-146{ }^{\circ} \mathrm{C}$, new compound, $R_{\mathrm{f}}=0.50$ (hexanes/ethyl acetate $=5: 1), 99 \%$ ee, $[\alpha]^{20} \mathrm{D}=-92.99(c 0.40$, EtOAc). ${ }^{1} \mathrm{H}$ NMR $\left(400 \mathrm{MHz}, \mathrm{CDCl}_{3}\right) \delta 7.93(\mathrm{~s}, 1 \mathrm{H}), 7.86(\mathrm{~d}, J=8.5$ $\mathrm{Hz}, 2 \mathrm{H}), 7.75$ (d, $J=8.2 \mathrm{~Hz}, 1 \mathrm{H}), 7.55(\mathrm{~d}, J=8.3 \mathrm{~Hz}, 2 \mathrm{H})$, 7.52-7.42 (m, 2H), 7.41-7.35 (m, 1H), 7.33-7.27 (m, 2H), $7.25-7.12(\mathrm{~m}, 3 \mathrm{H}), 7.04(\mathrm{~d}, J=7.8 \mathrm{~Hz}, 1 \mathrm{H}), 6.99(\mathrm{~d}, J=7.0 \mathrm{~Hz}$, $1 \mathrm{H}), 6.93(\mathrm{t}, J=7.7 \mathrm{~Hz}, 3 \mathrm{H}), 6.57(\mathrm{~d}, J=1.8 \mathrm{~Hz}, 1 \mathrm{H}), 6.35(\mathrm{~s}, 1 \mathrm{H})$, $6.00(\mathrm{~s}, 1 \mathrm{H}), 2.46(\mathrm{~s}, 3 \mathrm{H}), 2.16(\mathrm{~s}, 3 \mathrm{H}) ;{ }^{13} \mathrm{C} \mathrm{NMR}(100 \mathrm{MHz}$, $\left.\mathrm{CDCl}_{3}\right) \delta 154.4,153.4,144.0,136.4,136.1,136.0,134.0,131.4$, 129.6, 128.9, 127.9, 127.6, 126.4, 126.4, 126.4, 126.0, 125.7, 125.6, 124.5, 124.1, 123.7, 123.3, 123.2, 120.6, 120.3, 120.1, 116.8, 115.6, 114.0, 111.6, 36.8, 21.6, 16.7. HPLC: Chiralcel IC column, $254 \mathrm{~nm}, 30{ }^{\circ} \mathrm{C}, n$-hexane $/ i-\mathrm{PrOH}=90 / 10$, flow $=0.7$ $\mathrm{mL} / \mathrm{min}$, retention time $18.0 \mathrm{~min}$ (major) and $21.1 \mathrm{~min}$. HRMS calculated for $\mathrm{C}_{35} \mathrm{H}_{32} \mathrm{~N}_{3} \mathrm{O}_{3} \mathrm{~S} \quad\left[\mathrm{M}+\mathrm{NH}_{4}\right]^{+}$574.2159, found: 574.2161.

(+)- $N$-(2-((4-Chlorophenyl)(7-methyl-1H-indol-3-yl)methyl Jbenzofuran-3-yl)-4-methylbenzenesulfonamide (3jh): $100 \mathrm{mg}$, $92 \%$ yield, pink solid, m.p. $=124-126^{\circ} \mathrm{C}$, new compound, $R_{\mathrm{f}}=$ 0.45 (hexanes/ethyl acetate $=5: 1), 98 \%$ ee, $[\alpha]^{20} \mathrm{D}=+102.66(c$ 0.30, EtOAc). ${ }^{1} \mathrm{H}$ NMR (400 MHz, $\left.\mathrm{CDCl}_{3}\right) \delta 7.96(\mathrm{~s}, 1 \mathrm{H}), 7.60(\mathrm{~d}, J$ $=7.3 \mathrm{~Hz}, 2 \mathrm{H}), 7.35(\mathrm{~d}, J=8.4 \mathrm{~Hz}, 1 \mathrm{H}), 7.24-7.15(\mathrm{~m}, 4 \mathrm{H})$, 7.13-6.93 (m, 8H), $6.85(\mathrm{~s}, 1 \mathrm{H}), 6.26(\mathrm{~s}, 1 \mathrm{H}), 5.69(\mathrm{~s}, 1 \mathrm{H}), 2.47$ (s, 3H), 2.24 (s, 3H); ${ }^{13} \mathrm{C}$ NMR $\left(100 \mathrm{MHz}, \mathrm{CDCl}_{3}\right) \delta 155.5,153.4$, 144.2, 138.8, 136.4, 136.0, 132.7, 130.0, 129.7, 128.6, 127.7, $126.0,126.0,124.6,123.3,123.2,123.2,120.5,120.3,119.5$, 117.2, 115.6, 113.1, 111.7, 39.2, 21.5, 16.7. HPLC: Chiralcel $\mathrm{AD}-\mathrm{H}$ column, $254 \mathrm{~nm}, 30^{\circ} \mathrm{C}, n$-hexane $/ i$-PrOH $=75 / 25$, flow $=$ $0.8 \mathrm{~mL} / \mathrm{min}$, retention time $29.1 \mathrm{~min}$ and $31.4 \mathrm{~min}$ (major). HRMS calculated for $\mathrm{C}_{31} \mathrm{H}_{29} \mathrm{ClN}_{3} \mathrm{O}_{3} \mathrm{~S}\left[\mathrm{M}+\mathrm{NH}_{4}\right]^{+} 558.1613$, found: 558.1614.

(+)-4-Methyl- $N$-(2-((1-methyl- $1 H$-indol-3-yl)(phenyl)methy l)benzofuran-3-yl)benzenesulfonamide (3ai): $50 \mathrm{mg}, \quad 49 \%$ yield, white solid, m.p. $=229-231{ }^{\circ} \mathrm{C}$, new compound, $R_{\mathrm{f}}=0.20$ (hexanes/ethyl acetate $=10: 1), 49 \%$ ee, $[\alpha]^{20} \mathrm{D}=+54.25(c 0.40$, EtOAc). ${ }^{1} \mathrm{H}$ NMR (400 MHz, DMSO-d $) \delta 10.08(\mathrm{~s}, 1 \mathrm{H}), 7.54(\mathrm{~d}, J$ $=8.2 \mathrm{~Hz}, 2 \mathrm{H}), 7.44(\mathrm{~d}, J=8.1 \mathrm{~Hz}, 1 \mathrm{H}), 7.36(\mathrm{~d}, J=8.2 \mathrm{~Hz}, 1 \mathrm{H})$, 7.32 (d, $J=7.7 \mathrm{~Hz}, 1 \mathrm{H}), 7.28-7.08(\mathrm{~m}, 9 \mathrm{H}), 6.99-6.87(\mathrm{~m}, 3 \mathrm{H})$, $6.84(\mathrm{~s}, 1 \mathrm{H}), 5.81(\mathrm{~s}, 1 \mathrm{H}), 3.69(\mathrm{~s}, 3 \mathrm{H}), 2.05(\mathrm{~s}, 3 \mathrm{H}) ;{ }^{13} \mathrm{C} \mathrm{NMR}$ $(100 \mathrm{MHz}$, DMSO-d6) $\delta 154.5,152.7,142.9,140.8,136.9,136.6$, $129.2,128.3,128.1,127.9,126.7,126.5,126.5,125.9,124.2$, 122.8, 121.1, 119.9, 119.0, 118.6, 113.3, 113.1, 111.3, 109.6, 38.4, 32.2, 20.8. HPLC: Chiralcel IC column, $254 \mathrm{~nm}, 30^{\circ} \mathrm{C}$, $n$-hexane $/ i-\mathrm{PrOH}=90 / 10$, flow $=0.7 \mathrm{~mL} / \mathrm{min}$, retention time $25.1 \mathrm{~min}$ and $28.0 \mathrm{~min}$ (major). HRMS calculated for $\mathrm{C}_{31} \mathrm{H}_{26} \mathrm{~N}_{2} \mathrm{NaO}_{3} \mathrm{~S}[\mathrm{M}+\mathrm{Na}]+529.1556$, found: 529.1554 . 
4-Methyl- $N$-(2-(phenyl(1H-pyrrol-3-yl)methyl)benzofuran3-yl)benzenesulfonamide (3aj): $55 \mathrm{mg}, 62 \%$ yield, pink solid, m.p. $=256-257{ }^{\circ} \mathrm{C}$, new compound, $R_{\mathrm{f}}=0.30$ (hexanes/ethyl acetate $=10: 1), 1 \%$ ee. ${ }^{1} \mathrm{H} \mathrm{NMR}\left(400 \mathrm{MHz}, \mathrm{CDCl}_{3}\right) \delta 8.62(\mathrm{~s}, 1 \mathrm{H})$, $7.62(\mathrm{~d}, J=8.3 \mathrm{~Hz}, 2 \mathrm{H}), 7.34(\mathrm{~d}, J=8.3 \mathrm{~Hz}, 1 \mathrm{H}), 7.29-7.25(\mathrm{~m}$, 1H), 7.25-7.21 (m, 4H), 7.19-7.09 (m, 3H), $6.97(\mathrm{t}, J=7.5 \mathrm{~Hz}$, $1 \mathrm{H}), 6.78(\mathrm{~d}, J=7.7 \mathrm{~Hz}, 1 \mathrm{H}), 6.76-6.67(\mathrm{~m}, 1 \mathrm{H}), 6.19-6.02(\mathrm{~m}$, 2H), $5.91(\mathrm{~s}, 1 \mathrm{H}), 5.76(\mathrm{~s}, 1 \mathrm{H}), 2.37(\mathrm{~s}, 3 \mathrm{H}) ;{ }^{13} \mathrm{C}$ NMR $(100 \mathrm{MHz}$, $\left.\mathrm{CDCl}_{3}\right) \delta 155.6,153.4,144.4,139.0,136.5,130.0,129.9,128.7$, 128.7, 127.7, 127.4, 125.5, 124.7, 123.1, 118.9, 118.1, 113.3, 111.9, 108.3, 107.9, 41.4, 21.7. HPLC: Chiralcel IC column, 254 $\mathrm{nm}, 30{ }^{\circ} \mathrm{C}, n$-hexane $/ i$-PrOH $=70 / 30$, flow $=0.7 \mathrm{~mL} / \mathrm{min}$, retention time $6.9 \mathrm{~min}$ (major) and $7.6 \mathrm{~min}$. HRMS calculated for $\mathrm{C}_{26} \mathrm{H}_{23} \mathrm{~N}_{2} \mathrm{O}_{3} \mathrm{~S}[\mathrm{M}+\mathrm{H}]+443.1424$, found: 443.1421 .

\subsection{Experiment at gram scale}

To a solution of azadiene 1a (939 mg, $2.5 \mathrm{mmol}$ ) and chiral phosphoric acid (R)-TRIP (A1) (90.7 $\mathrm{mg}, 0.125 \mathrm{mmol}$ ) in mesitylene (37.5 mL) at $-20{ }^{\circ} \mathrm{C}$, indole $2 \mathrm{a}(292 \mathrm{mg}, 2.5 \mathrm{mmol})$ was added. The reaction was stirred at $-20{ }^{\circ} \mathrm{C}$ for $48 \mathrm{~h}$. The crude product was directly purified by silica gel column chromatography (eluent: hexanes/ethyl acetate $=10: 1$ to $5: 1$ ) to give the desirable chiral hetero-triarylmethane 3aa, $1.166 \mathrm{~g}$, 95\% yield, 90\% ee. HPLC: Chiralcel IC column, $254 \mathrm{~nm}, 30{ }^{\circ} \mathrm{C}$, $n$-hexane $/ i$-PrOH $=90 / 10$, flow $=0.7 \mathrm{~mL} / \mathrm{min}$, retention time 20.7 min (major) and $23.3 \mathrm{~min}$ (minor).

\subsection{Synthetic transformation of compound (-)-3fh}

Under nitrogen atmosphere, to a solution of (-)-3fh $(99 \%$ ee, $55 \mathrm{mg}, 0.10 \mathrm{mmol}$ ) in anhydrous tetrahydrofuran, triethylamine $\quad(12.2 \quad \mathrm{mg}, \quad 0.12 \quad \mathrm{mmol}) \quad$ and 4-dimethylaminopyridine (DMAP, $12.2 \mathrm{mg}, 0.10 \mathrm{mmol}$ ) were added in sequence at $0{ }^{\circ} \mathrm{C}$, and then $\mathrm{TsCl}(23.0 \mathrm{mg}, 0.12 \mathrm{mmol})$ in tetrahydrofuran was added dropwise. The reaction was monitored by thin-layer chromatography. After the starting material disppeared, the solvent was removed under reduced pressure. Then water was added and the mixture was extracted with dichloromethane, The combined organic layer was washed with water and brine, dried over anhydrous sodium sulfate, and concentrated under reduced pressure. The crude material was purified by silica gel column chromatography (dichloromethane/hexanes $1 / 2$ to $1 / 1$ ) to give the product (-)-4 (60 mg, 85\%) as white solid.

(S)-(-)-4-Methyl- $N$-(2-((7-methyl-1H-indol-3-yl)(naphthale n-1-yl)methyl)benzofuran-3-yl)- $N$-tosylbenzenesulfonamide

(4): $60 \mathrm{mg}, 85 \%$ yield, white solid, m.p. $=260-261{ }^{\circ} \mathrm{C}$, new compound, $R_{\mathrm{f}}=0.60$ (hexanes/ethyl acetate $=5: 1$ ), $99 \%$ ee, $[\alpha]^{20} \mathrm{D}=-244.58\left(c 0.50, \mathrm{CH}_{2} \mathrm{Cl}_{2}\right) .{ }^{1} \mathrm{H}$ NMR $\left(400 \mathrm{MHz}, \mathrm{CDCl}_{3}\right) \delta$ $8.07(\mathrm{~d}, J=8.5 \mathrm{~Hz}, 1 \mathrm{H}), 7.96(\mathrm{~s}, 1 \mathrm{H}), 7.92(\mathrm{~d}, J=7.5 \mathrm{~Hz}, 1 \mathrm{H}), 7.85$ (d, $J=8.1 \mathrm{~Hz}, 1 \mathrm{H}), 7.76(\mathrm{~d}, J=8.4 \mathrm{~Hz}, 2 \mathrm{H}), 7.68-7.59(\mathrm{~m}, 1 \mathrm{H})$, 7.54-7.41 (m, 2H), 7.39-7.32 (m, 3H), 7.19-7.12 (m, 3H), 7.09 $(\mathrm{d}, J=8.2 \mathrm{~Hz}, 2 \mathrm{H}), 7.04-6.91(\mathrm{~m}, 4 \mathrm{H}), 6.74(\mathrm{~s}, 1 \mathrm{H}), 6.62(\mathrm{~d}, J=$ $7.8 \mathrm{~Hz}, 1 \mathrm{H}), 6.46(\mathrm{~d}, J=8.2 \mathrm{~Hz}, 2 \mathrm{H}), 2.46(\mathrm{~s}, 3 \mathrm{H}), 2.38(\mathrm{~s}, 3 \mathrm{H})$, 2.09 (s, 3H); ${ }^{13} \mathrm{C}$ NMR $\left(100 \mathrm{MHz}, \mathrm{CDCl}_{3}\right) \delta 160.6,153.2,145.4$, 144.6, 137.7, 136.3, 135.9, 135.9, 134.1, 131.7, 129.5, 129.4,
129.0, 128.7, 128.5, 127.9, 126.6, 126.5, 126.4, 126.3, 125.7, 125.7, 124.9, 124.7, 124.3, 123.3, 122.7, 120.2, 120.0, 119.4, 118.1, 116.1, 112.7, 112.0, 36.9, 21.8, 21.5, 16.7. HPLC: chiralcel $\mathrm{AD}-\mathrm{H}$ column, $254 \mathrm{~nm}, 30^{\circ} \mathrm{C}, n$-hexane $/ i$-PrOH $=80 / 20$, flow $=$ $0.7 \mathrm{~mL} / \mathrm{min}$, retention time $19.3 \mathrm{~min}$ and $25.4 \mathrm{~min}$ (major). HRMS calculated for $\mathrm{C}_{42} \mathrm{H}_{38} \mathrm{~N}_{3} \mathrm{O}_{5} \mathrm{~S}_{2}\left[\mathrm{M}+\mathrm{NH}_{4}\right]+728.2247$, found: 728.2257.

\section{Results and discussion}

At the outset, azadiene 1a and indole 2a were chosen as model substrates for condition optimization. To our delight, the reaction proceeded smoothly in $10 \mathrm{~min}$ to give the anticipated product with $90 \%$ isolated yield and $67 \%$ ee by employing chiral BINOL-based phosphoric acid $(R)$-A1 as catalyst (Table 1, entry 1). A series of solvents were evaluated, and it was found that solvent played a crucial role in controlling the enantioselectivity of the reaction (entries 1-6). Polar solvents such as tetrahydrofuran exhibited poor enantioselectivity (entry 3). Mesitylene proved to be the most favourable solvent in view of reactivity and enantioselectivity (entry 6). Subsequently, various chiral phosphoric acids including BINOL and H8-BINOL skeletons were explored (entries 6-11). $(R)$-TRIP (A1) was the most efficient catalyst, providing the desired product 3aa with $81 \%$ ee (entry 6). To further improve the enantioselectivity, the effect of temperature was examined (entries 12 and 13). When the reaction temperature was decreased to $-20{ }^{\circ} \mathrm{C}$, a higher enantioselectivity (89\%) was obtained and the reactivity could be maintained by extending

Table 1

The evaluation of reaction parameters ${ }^{\text {a. }}$.

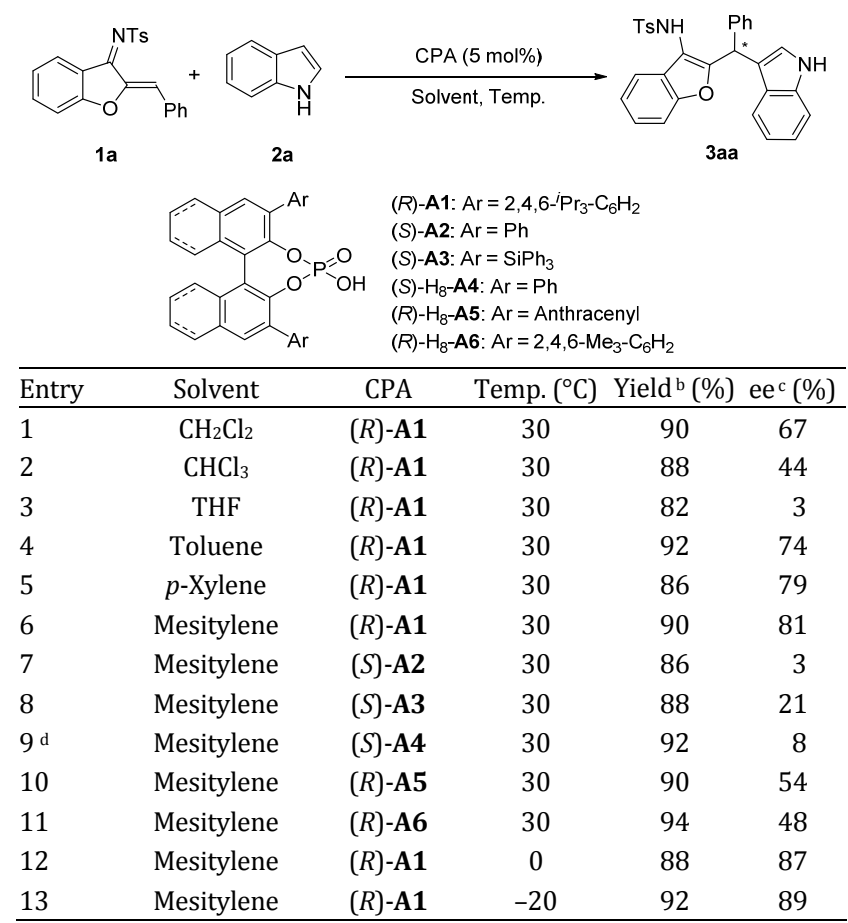

a Reaction conditions: azadiene 1a $(0.10 \mathrm{mmol})$, indole $2 \mathbf{a}(0.10 \mathrm{mmol})$, CPA (5 mol\%), solvent (1.5 mL).

b Isolated yield.

c Determined by HPLC. 
the reaction time to $48 \mathrm{~h}$ (entry 13). Therefore, the optimal condition was established: using $(R)$-TRIP as catalyst and mesitylene as solvent to perform the reaction at $-20{ }^{\circ} \mathrm{C}$. After establishing the optimal conditions, we examined the scope of conjugate addition of indoles $\mathbf{1}$ to azadienens $\mathbf{2}$ and the results are depicted in Scheme 2. In general, the reaction performed very well, delivering the corresponding chiral hetero-triarylmethanes in good yields and enantioselectivities. Various indoles were suitable for the conjugate addition. Steric properties of substituents on indole had an obvious influence on the enantioinduction. When a methyl group was introduced at the 2-position of the indole, the desired adduct $\mathbf{3 a b}$ was obtained in moderate enantioselectivity. Good enantioselectivities and yields were achieved for indoles with both electron-donating and electron-withdrawing substituents at the 5 -position. The reaction of 5-chloroindole with azadiene could provide adduct 3ad with $89 \%$ ee and $93 \%$ yield. It was worth noting that using 7-methylindole as nucleophile, the corresponding product 3ah was acquired in excellent enantioselec-

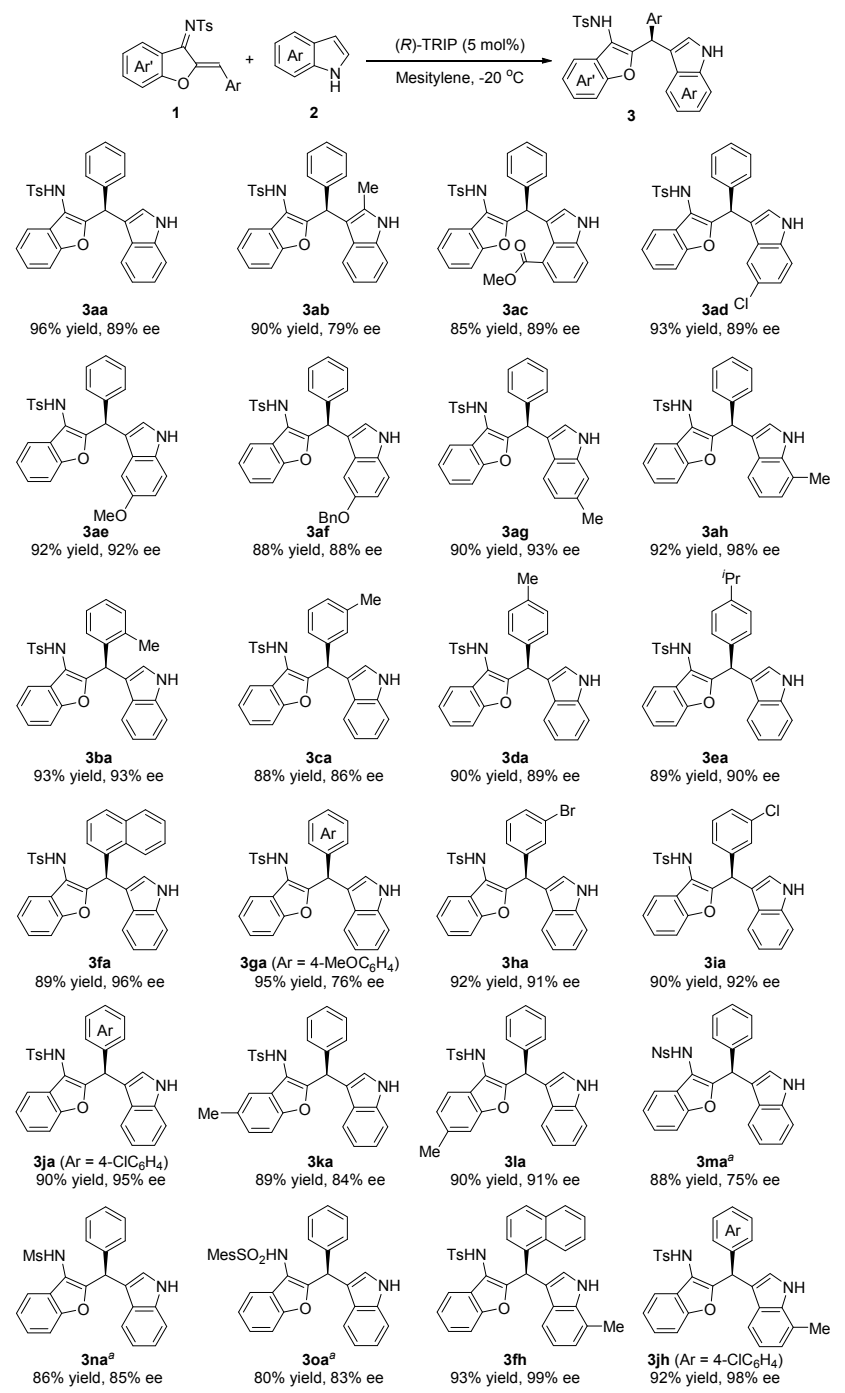

Scheme 2. Substrate scope. Conditions: azadienes $1(0.20 \mathrm{mmol})$, indoles 2 (0.20 mmol), (R)-TRIP (A1) (5 mol\%), mesitylene (3.0 mL), -20 ${ }^{\circ} \mathrm{C}, 48-72$ h. a $0{ }^{\circ} \mathrm{C}, 48 \mathrm{~h}$. tivity and yield. Further research was focused on a wide array of azadienes. The steric properties of the substituents on the aromatic ring had only marginal effect on yields and enantioselectivities. For example, the reaction furnished the target products $3 \mathbf{b a}$ and $3 \mathbf{d a}$ in $93 \%$ and $89 \%$ ee, respectively. However, probably owing to electronic effects, the introduction of electron-donating methoxy group at the para-position of the aromatic ring resulted in the decreased ee value, and moderate enantioselectivity of $\mathbf{3 g a}$ was delivered. The azadienes with methyl substituent at the 5- or 6-position of benzofuryl ring were also suitable reaction partners, giving the corresponding products 3ka and 3la in $84 \%$ and $91 \%$ ee, respectively. When the reaction temperature was increased to $0{ }^{\circ} \mathrm{C}$, sulfonylimines 3m-3o were transformed successfully with moderate to good enantioselectivities. Notably, the conjugate addition of azadienes 3f and 3j with 7-methylindole proceeded smoothly, achieving excellent enantioselectivities and yields. Moreover, the addition of pyrrole to azadiene performed well with high reactivity albeit with very low enantioselectivity $(1 \%$ ee) (see the Supporting Information for details).

In order to further demonstrate the practicality of this methodology, the conjugate addition of indole $\mathbf{2 a}$ to azadiene 1a was conducted at gram scale, giving the desired product in 95\% yield and $90 \%$ ee without noticeable loss of yield and enantioselectivity (Scheme 3).

The additional tosylation of hetero-triarylmethane (-)-3fh with $p$-tosyl chloride, delivering the $\mathrm{N}, \mathrm{N}$-bistosylamide $(-)-4$ with good yield and without loss of enantiopurity (Scheme 4). The absolute configuration of compound (-)-4, which was recrystallized from dichloromethane and $n$-hexane as a colorless crystal, was unambiguously determined to be $S$ by X-ray crystallographic analysis [59] (Scheme 4). Therefore, the absolute configuration of hetero-triarylmethane (-)-3fh was assigned as $(S)-(-)-\mathbf{3 f h}$.

We performed a control experiment to gain insight into the plausible mechanism. When $N$-methylindole $2 \mathbf{i}$ was used as nucleophile to react with azadiene 1a under the above standard conditions, the addition product 3ai was obtained in only $49 \%$ yield and $49 \%$ ee by extending the reaction time to $120 \mathrm{~h}$ (Eq. 1). This result suggested the free $\mathrm{N}-\mathrm{H}$ moiety of indole might provide a hydrogen-bonding interaction with the phos-

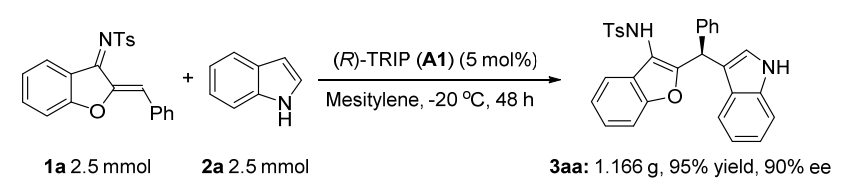

Scheme 3. Scale-up experiment.

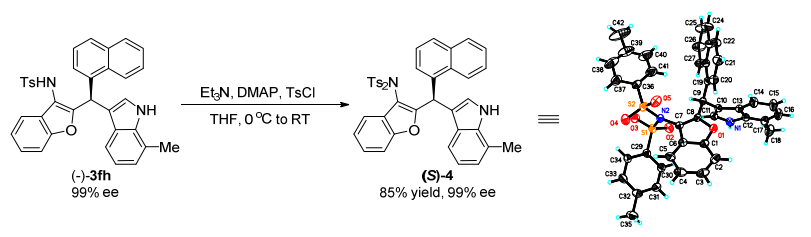

Scheme 4. Synthetic transformation of compound (-)-3fh and X-ray crystal structure of compound (-)-4. 


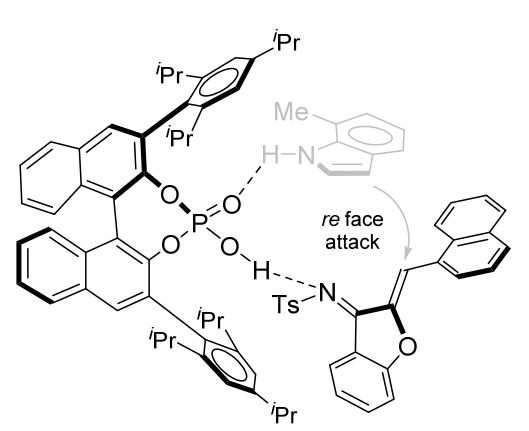

Fig. 1. A plausible transition state.

phoryl oxygen atom of chiral phosphoric acid catalyst.

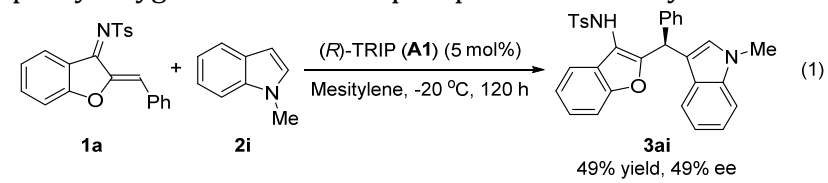

On the basis of the above experimental results, we proposed a plausible transition-state model to explain the absolute configuration structure of hetero-triarylmethane products (Fig. 1). The chiral phosphoric acid simultaneously activated azadiene and indole via dual hydrogen-bonding interaction. The triisopropyl phenyl groups at the 3,3'-positions of the catalyst shielded the si-face of azadiene, and the conjugate addition preferentially occurred at the re-face of azadiene, affording $S$-configured hetero-triarylmethane product.

\section{Conclusions}

We have successfully developed an efficient chiral Brønsted acid-catalyzed conjugate addition of indoles to azadienes for the preparation of structurally important optically active hetero-triarylmethanes with excellent enantioselectivities and broad substrate scope. This chiral Brønsted acid catalytic system provides a new opportunity for the development of asymmetric reactions of azadienes. Further explorations on the application of this strategy are ongoing in our laboratory.

\section{References}

[1] T. P. Pathak, M. S. Sigman, J. Org. Chem., 2011, 76, 9210-9215.

[2] W.-J. Bai, J. G. David, Z.-G. Feng, M. G. Weaver, K.-L. Wu, T. R. R. Pettus, Acc. Chem. Res., 2014, 47, 3655-3664.

[3] M. S. Singh, A. Nagaraju, N. Anand, S. Chowdhury, RSC Adv., 2014, 4, 55924-55959.

[4] Z. Wang, J. Sun, Synthesis, 2015, 47, 3629-3644.

[5] L. Caruana, M. Fochi, L. Bernardi, Molecules, 2015, 20, 11733-11764.

[6] A. A. Jaworski, K. A. Scheidt, J. Org. Chem., 2016, 81, 10145-10153.

[7] B. Yang, S. Gao, Chem. Soc. Rev., 2018, 47, 7926-7953.

[8] J. Chen, Y. Huang, Org. Lett., 2017, 19, 5609-5612.

[9] J. Chen, P. Jia, Y. Huang, Org. Lett., 2018, 20, 6715-6718.

[10] L.-C. Yang, Z.-Q. Rong, Y.-N. Wang, Z. Y. Tan, M. Wang, Y. Zhao, Angew. Chem. Int. Ed., 2017, 56, 2927-2931.

[11] Z.-Q. Rong, L.-C. Yang, S. Liu, Z. Yu, Y.-N. Wang, Z. Y. Tan, R.-Z. Huang, Y. Lan, Y. Zhao, J. Am. Chem. Soc., 2017, 139, 15304-15307.

[12] Y.-N. Wang, L.-C. Yang, Z.-Q. Rong, T.-L. Liu, R. Liu, Y. Zhao, Angew. Chem. Int. Ed., 2018, 57, 1596-1600.

[13] Z.-Q. Rong, M. Wang, C. H. E. Chow, Y. Zhao, Chem.-Eur. J., 2016, 22, 9483-9487.

[14] K.-Q. Chen, Z.-H. Gao, S. Ye, Org. Chem. Front, 2019, 6, 405-409.

[15] Z.-H. Gao, K.-Q. Chen, Y. Zhang, L.-M. Kong, Y. Li, S. Ye, J. Org. Chem., 2018, 83, 15225-15235.

[16] H. Ni, X. Tang, W. Zheng, W. Yao, N. Ullah, Y. Lu, Angew. Chem. Int. $E d ., 2017,56,14222-14226$.

[17] Z. Gu, J. Zhou, G.-F. Jiang, Y.-G. Zhou, Org. Chem. Front., 2018, 5, $1148-1151$.

[18] Z. Gu, J.-J. Xie, G.-F. Jiang, Y.-G. Zhou, Asian J. Org. Chem., 2018, 7, 1561-1564.

\section{Graphical Abstract}

Chin. J. Catal., 2019, 40: 1566-1575 doi: S1872-2067(19)63396-6

Chiral Brønsted acid-catalyzed conjugate addition of indoles to azadienes: Enantioselective synthesis of hetero-triarylmethanes

Huan-Ping Xie, Bo Wu, Xin-Wei Wang, Yong-Gui Zhou*

Dalian Institute of Chemical Physics, Chinese Academy of Sciences; University of Chinese Academy of Sciences

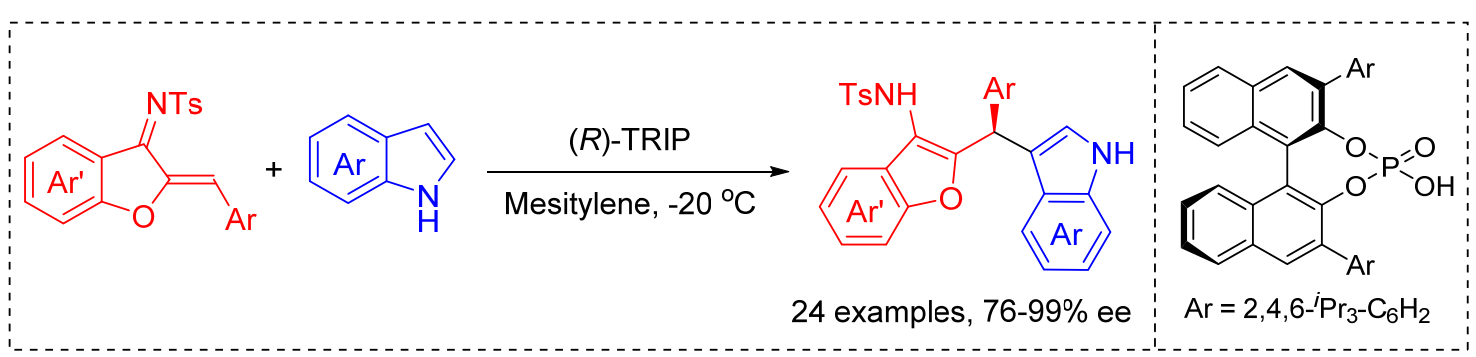

An efficient chiral Brønsted acid-catalyzed conjugate addition of indoles to azadienes has been successfully developed, which enables a facile approach to optically active hetero-triarylmethanes with excellent enantioselectivities and broad substrate scope. This chiral Brønsted acid catalytic system provides a new opportunity for the development of asymmetric reactions of azadienes. 
[19] W. Lin, C. Zhang, W. Xu, Y. Cheng, P. Li, W. Li, Adv. Synth. Catal., 2019, 361, 476-480.

[20] Z. Gu, B. Wu, G.-F. Jiang, Y.-G. Zhou, Chin. J. Chem., 2018, 36, 1130-1134.

[21] T. Akiyama, Chem. Rev., 2007, 107, 5744-5758.

[22] S.-L. You, Q. Cai, M. Zeng, Chem. Soc. Rev., 2009, 38, 2190-2201.

[23] M.Zeng, S.-L. You, Synlett, 2010, 1289-1301.

[24] D. Kampen, C. M. Reisinger, B. List, Top. Curr. Chem., 2010, 291, 395-456.

[25] M. Terada, Synthesis, 2010, 1929-1982.

[26] A. Zamfir, S. Schenker, M. Freund, S. B. Tsogoeva, Org. Biomol. Chem., 2010, 8, 5262-5276.

[27] M. Rueping, A. Kuenkel, I. Atodiresei, Chem. Soc. Rev., 2011, 40, 4539-4549.

[28] S. Schenker, A. Zamfir, M. Freund, S. B. Tsogoeva, Eur. J. Org. Chem., 2011, 2209-2222.

[29] M. Terada, Curr. Org. Chem., 2011, 15, 2227-2256.

[30] R. J. Phipps, G. L. Hamilton, F. D. Toste, Nat. Chem., 2012, 4, 603-614.

[31] M. Mahlau, B. List, Angew. Chem. Int. Ed., 2013, 52, 518-533.

[32] D. Parmar, E. Sugiono, S. Raja, M. Rueping, Chem. Rev., 2014, 114, 9047-9153.

[33] F.-L. Sun, M. Zeng, Q. Gu, S.-L. You, Chem.-Eur. J., 2009, 15, 8709-8712.

[34] F.-L. Sun, X.-J. Zheng, Q. Gu, Q.-L. He, S.-L. You, Eur. J. Org. Chem., 2010, 47-50.

[35] M.-H. Zhuo, Y.-J. Jiang, Y.-S. Fan, Y. Gao, S. Liu, S. Zhang, Org. Lett., 2014, 16, 1096-1099.

[36] S. Qi, C.-Y. Liu, J.-Y. Ding, F.-S. Han, Chem. Commun., 2014, 50, 8605-8608.

[37] Y.-X. Gong, Q. Wu, H.-H. Zhang, Q.-N. Zhu, F. Shi, Org. Biomol. Chem., 2015, 13, 7993-8000.

[38] S. Saha, S. K. Alamsetti, C. Schneider, Chem. Commun., 2015, 51, 1461-1464.

[39] W. Zhao, Z. Wang, B. Chu, J. Sun, Angew. Chem. Int. Ed., 2015, 54, 1910-1913.

[40] Z. Wang, Y. F. Wong, J. Sun, Angew. Chem. Int. Ed., 2015, 54, 13711-13714.

[41] H.-H. Liao, A. Chatupheeraphat, C.-C. Hsiao, I. Atodiresei, M. Rueping, Angew. Chem. Int. Ed., 2015, 54, 15540-15544.

[42] J. Zheng, L. Lin, L. Dai, X. Yuan, X. Liu, X. Feng, Chem.-Eur. J., 2016,
22, 18254-18258.

[43] M.-H. Zhuo, G.-F. Liu, S.-L. Song, D. An, J. Gao, L. Zheng, S. Zhang, Adv. Synth. Catal., 2016, 358, 808-815.

[44] Y. Zhang, S.-X. Zhang, L.-N. Fu, Q.-X. Guo, ChemCatChem, 2017, 9, 3107-3110.

[45] C. Yue, F. Na, X. Fang, Y. Cao, J. C. Antilla, Angew. Chem. Int. Ed., 2018, 57, 11004-11008.

[46] P. Finocchiaro, D. Gust, K. Mislow, J. Am. Chem. Soc., 1974, 96, 3198-3205.

[47] H. V. Bossche, G. Willemsens, I. Roels, D. Bellens, H. Moereels, M. C. Coene, L. L. Jeune, W. Lauwers, P. A. J. Janssen, Biochem. Pharmacol., 1990, 40, 1707-1718.

[48] H. W. Gibson, S. H. Lee, P. T. Engen, P. Lecavalier, J. Sze, Y. X. Shen, M. Bheda, J. Org. Chem., 1993, 58, 3748-3756.

[49] G. Panda, Shagufta, J. K. Mishra, V. Chaturvedi, A. K. Srivastava, R. Srivastava, B. S. Srivastava, Bioorg. Med. Chem., 2004, 12, 5269-5276.

[50] R. S. Dothager, K. S. Putt, B. J. Allen, B. J. Leslie, V. Nesterenko, P. J. Hergenrother, J. Am. Chem. Soc., 2005, 127, 8686-8696.

[51] V. Nair, S. Thomas, S. C. Mathew, K. G. Abhilash, Tetrahedron, 2006, 62, 6731-6747.

[52] S. Gemma, G. Campiani, S. Butini, G. Kukreja, B. P. Joshi, M. Persico, B. Catalanotti, E. Novellino, E. Fattorusso, V. Nacci, L. Savini, D. Taramelli, N. Basilico, G. Morace, V. Yardley, C. Fattorusso, J. Med. Chem., 2007, 50, 595-598.

[53] S. D. Cho, K. Yoon, S. Chintharlapalli, M. Abdelrahim, P. Lei, S. Hamilton, S. Khan, S. K. Ramaiah, S. Safe, Cancer Res., 2007, 67, 674-683.

[54] R. Palchaudhuri, V. Nesterenko, P. J. Hergenrother, J. Am. Chem. Soc., 2008, 130, 10274-10281.

[55] M. K. Parai, G. Panda, V. Chaturvedi, Y. K. Manju, S. Sinha, Bioorg. Med. Chem. Lett., 2008, 18, 289-292.

[56] S. A. Snyder, S. P. Breazzano, A. G. Ross, Y. Lin, A. L. Zografos, J. Am. Chem. Soc., 2009, 131, 1753-1765.

[57] M. Shiri, M. A. Zolfigol, H. G. Kruger, Z. Tanbakouchian, Chem. Rev., 2010, 110, 2250-2293.

[58] X. Li, S. O. Lee, S. Stephen, Biochem. Pharmacol., 2012, 83, 1445-1455.

[59] CCDC number is 1878318. These details can be obtained free of charge via www.ccdc.com.ac.uk/data_request/cif from the Cambridge Crystallographic Data Centre.

\title{
手性布朗斯特酸催化吲哚与氮杂二烯的共轭加成反应对映选择性合成 杂三芳基甲烷
}

\author{
谢焕平 $\mathrm{a}, \mathrm{b}$ ，吴 波 ${ }^{\mathrm{a}}$, 王新维 ${ }^{\mathrm{a}}$ ，周永贵 $\mathrm{a}^{\mathrm{a}}$ * \\ ${ }^{\mathrm{a}}$ 中国科学院大连化学物理研究所催化基础国家重点实验室, 辽宁大连116023 \\ 中国科学院大学, 北京 100049
}

摘要: 呋喃酮衍生的氮杂二烯具有恢复芳香性的特点, 是一类重要的高活性中间体. 近年来, 呋喃酮衍生的氮杂二烯的不 对称催化反应已经取得重要进展, 并且发展了多种有效的催化体系, 包括过渡金属催化体系、手性胺催化体系、氮杂环卡 宾催化体系、手性膦催化体系以及手性布朗斯特碱催化体系. 这些催化体系丰富了氮杂二烯的不对称反应类型如亲核加 成和环合反应, 同时为具有生物活性结构单元的合成提供了新的途径. 尽管在有机催化中手性布朗斯特酸是一类非常重 要的催化剂, 已成功应用于不对称催化反应中, 然而手性布朗斯特酸在氮杂二烯中间体不对称化学中的应用却未见报道. 为了进一步丰富氮杂二烯的不对称反应类型和构建更多的具有生物活性的结构单元, 发展新的催化体系应用于氮杂二烯 的不对称反应具有重要意义.

基于本课题组之前对氮杂二烯不对称催化反应的研究, 本文发展了一种手性布朗斯特酸催化吲哚与氮杂二烯的共轭 
加成反应对映选择性合成杂三芳基甲烷的方法. 通过对催化剂、溶剂和温度的篎选, 得到了最优反应条件: 使用在 3,3'-位 引入大位阻的2,4,6-三异丙基苯基取代的BINOL 衍生的手性磷酸作为催化剂, 均三甲苯为溶剂, 反应温度为-20 ${ }^{\circ} \mathrm{C}$. 该反应 具有较好的普适性, 共合成了 24 个手性杂三芳基甲烷化合物, 分离收率是 $80 \%-96 \%$, 最高对映选择性可达 $99 \%$. 为了提高 该合成方法的实用性, 进行了克级规模反应. 实验结果表明, 氮杂二烯和吲哚的用量由 $0.20 \mathrm{mmol}$ 增加至 $2.5 \mathrm{mmol}$ 时, 不对 称共轭加成反应仍能以优秀的对映选择性(90\%)和收率(95\%)得到目标产物, 对映选择性可以保持.

总之, 我们采用手性磷酸作为有机催化剂成功实现了吲哚与氮杂二烯的高对映选择性共轭加成反应, 合成了一系列光 学活性的杂三芳基甲烷化合物, 为手性杂三芳基甲烷化合物的合成提供了一种新的有效方法, 为新药的开发奠定了基础. 该反应操作简单、条件温和并且底物适用范围广. 手性布朗斯特酸催化体系为氮杂二烯不对称催化反应的发展提供了新 的机会.

关键词: 共轭加成; 杂三芳基甲烷; 氮杂二烯; 吲哚; 手性布朗斯特酸

收稿日期: 2019-06-27. 接受日期: 2019-07-20. 出版日期: 2019-10-05.

*通讯联系人. 电话/传真: (0411)84379220; 电子信箱: ygzhou@dicp.ac.cn

基金来源：国家自然科学基金(21532006); 大连市科学技术局(2016RD07); 中国科学院战略性先导科技专项(XDB17020300).

本文的电子版全文由Elsevier出版社在ScienceDirect上出版(http://www.sciencedirect.com/science/journal/18722067). 